\title{
GENERALIZED EIGENFUNCTIONS AND COMPLETE SEMISEPARABLE SOLUTIONS FOR STOKES FLOW IN SPHEROIDAL COORDINATES
}

\author{
BY \\ G. DASSIOS, M. HADJINICOLAOU, ANd A. C. PAYATAKES \\ University of Patras and ICE/HT-FORTH, Greece
}

\begin{abstract}
The stream function $\psi$ for axisymmetric Stokes flow satisfies the wellknown equation $E^{4} \psi=0$. In spheroidal coordinates the equation $E^{2} \psi=0$ admits separable solutions in the form of products of Gegenbauer functions of the first and second kind, and the general solution is then represented as a series expansion in terms of these eigenfunctions. Unfortunately, this property of separability is not preserved when one seeks solutions of the equation $E^{4} \psi=0$. The nonseparability of $E^{4} \psi=0$ in spheroidal coordinates has impeded considerably the development of theoretical models involving particle-fluid interactions around spheroidal objects. In the present work the complete solution for $\psi$ in spheroidal coordinates is obtained as follows. First, the generalized 0-eigenspace of the operator $E^{2}$ is investigated and a complete set of generalized eigenfunctions is given in closed form, in terms of products of Gegenbauer functions with mixed order. The general Stokes stream function is then represented as the sum of two functions: one from the 0 -eigenspace and one from the generalized 0 -eigenspace of the operator $E^{2}$. A rearrangement of the complete expansion, in such a way that the angular-type dependence enters through the Gegenbauer functions of successive order, leads to some kind of semiseparable solutions, which are given in terms of full series expansions. The proper solution subspace that provides velocity and vorticity fields, which are regular on the axis, is given explicitly. Finally, it is shown how these simple and generalized eigenfunctions reduce to the corresponding spherical eigenfunctions as the focal distance of the spheroidal system tends to zero, in which case the separability is regained. The usefulness of the method is demonstrated by solving the problem of the flow in a fluid cell contained between two confocal spheroidal surfaces with Kuwabara-type boundary conditions.
\end{abstract}

Received March 18, 1992.

1991 Mathematics Subject Classification. Primary 76D07; Secondary 33A50, 33A70, 35C10, 35D99, $35 \mathrm{~J} 30$.

Key words and phrases. Stokes flow, generalized eigenfunctions, semiseparable solutions, creeping flow, low Reynolds number, spheroidal obstacles, spheroid-in-cell.

This work was supported by EC, DG XII, Program BRITE, Project P-2289, Contract No. RI1B-0290-C (AM), and by the Institute of Chemical Engineering and High Temperature Chemical Processes (ICE/HTFORTH). 
1. Introduction. Particle-fluid systems are encountered in many important applications, such as settling, flow in granular porous media, fluidization, hydraulic or pneumatic transport, crystallization, filtration, hydrodynamic chromatography, flow of emulsions, colloids, suspensions of living cells, etc. The particles in these systems are usually considered as spherical. Still, in many cases the particles (grains, crystals, agglomerates, macromolecules, bacteria, etc.) can be considered, more appropriately, as prolate or oblate spheroids. For this reason the family of problems of flow around spheroids, with diverse boundary conditions, is of considerable practical and theoretical interest. Here we are interested in Stokes flows, a condition that is satisfied in many cases because of the small size of the particles.

The equation of motion for axisymmetric Stokes flow, in terms of the Stokes stream function $\psi$, is $E^{4} \psi=0$, where $E^{4}$ is the well-known operator [7]. This equation is separable in Cartesian, cylindrical, and spherical coordinates. Furthermore, it is $R$-separable in bispherical coordinates (Stimson and Jeffery (1926) [20]; Morse and Feshbach (1953) [14]). Unfortunately, it is not separable in spheroidal coordinates, a fact that seems to have impeded considerably the development of analyses of particle-fluid interactions involving spheroidal objects, despite the inherent usefulness that these would have.

Stokes flow around spheroids and ellipsoids attracted the interest of researchers early. Oberbeck (1876) [15] obtained a solution in Cartesian coordinates for the Stokes flow caused by the steady translation of an ellipsoid in an unbounded fluid (see also Lamb (1932) [12]; Happel and Brenner (1965) [9]). To this end, Oberbeck used an ingenious method, based on Dirichlet's formula for the gravitational potential of the ellipsoid, assuming unit density.

In an elaborate work Sampson (1891) [19] presented among others an analysis of Stokes flow around a spheroid translating along its main axis in an unbounded fluid by employing spheroidal coordinates. Some of the results obtained in the present work can be found implicitly in Sampson's analysis. However, Sampson did not discover the existence of the generalized eigenfunctions, and thus his results were cast in a form that was not very useful for applications. This statement is not meant as a criticism of Sampson's work. In developing our solution we had the benefit of modern linear functional analysis, which was developed mainly during the past few decades.

The solution in spheroidal coordinates of the problem of axisymmetric flow around a single spheroid translating along its axis in an unbounded fluid was obtained by Payne and Pell (1960) [16]. These authors recognized that their solution could be obtained by appropriate simplification and change of coordinates from Oberbeck's (1876) solution for the ellipsoid. Happel and Brenner [9] also obtained the solution of this problem in spheroidal coordinates, employing an effective ad hoc technique to overcome the difficulty presented by the fact that the equation $E^{4} \psi=0$ is not separable, in its generality, in this system of coordinates.

Here, we derive a complete set of generalized eigenfunctions. Each eigenfunction is given in closed form, in terms of Gegenbauer functions of mixed order. Graphs of the streamlines generated by the first few generalized eigenfunctions are given. A 
rearrangement of the complete expansion in such a way that the angular-type dependence enters through the Gegenbauer functions of successive order produces some kind of semiseparable solution, which is given in terms of a full series expansion. The semiseparable solution is sufficiently general to handle interior, exterior, and spheroidal cell problems, with or without prescribed singularities on the axis of symmetry.

In order to demonstrate the usefulness of the method of semiseparation of variables we use it to determine Stokes flow in a spheroid-in-cell, as a model of flow through a swarm of spheroids. This application is a simple example of a problem that cannot be solved by an ad hoc technique, but requires use of the complete semiseparable solution developed here, because the subspace of separable solutions is too "small" to host the actual solution. This solution is utilized [6] to model flow through a swarm of spheroids and to obtain such useful engineering properties as the drag coefficient and the permeability.

Before proceeding with the determination of the generalized eigenfunctions and the development of the complete semiseparable solution, we should mention that several problems of Stokes flow around a single spheroid (or other slender body) have been successfully treated with the method of singularities. Exact solutions of Stokes flow around slender bodies in unbounded fluids by superposition of hydrodynamic singularities were developed by Chwang and Wu [2-4] and Chwang [1]. Rallison and Acrivos [18] developed the method of boundary integrals of distributed singularities. Jeffrey and Onishi [10] used multipoles and moment matching to obtain a solution of Stokes flow around two unequal rigid spheres immersed in a rather general flow field, even when the spheres are virtually in contact.

Recently, singularity methods were developed to deal with cases involving wall effects. Dabros [5] developed a method of discrete singularity placement that can be used to account for wall effects. Yoon and Kim [21] used multipoles and a boundary collocation method to calculate the relative motion of two spheroids moving in Stokes flow, taking into account the hydrodynamic interaction between the two bodies.

Analyses with the method of hydrodynamic singularities are fundamentally different in approach from the present work. The singularity representation of the generalized eigenfunctions obtained herein is the subject of current work.

2. Stokes flow. Our goal is to derive a complete representation of the Stokes stream function in prolate spheroidal coordinates. The solution should be sufficiently general to solve interior, exterior, and spheroidal shell problems, with or without prescribed singularities on the axis of symmetry. We assume that the flow is axisymmetric.

For convenience, the basic characteristics of the spheroidal geometry are summarized in Appendix A.

We look for a velocity field in the form

$$
\mathbf{v}(\tau, \zeta)=v_{\tau}(\tau, \zeta) \hat{\boldsymbol{\tau}}+v_{\zeta}(\tau, \zeta) \hat{\zeta}
$$

where $\hat{\boldsymbol{\tau}}, \hat{\boldsymbol{\zeta}}$ denote the meridian unit vectors of the spheroidal system. Here we have 
used the transformation

$$
\begin{array}{llrl}
\tau=\cosh \eta, & \frac{\partial}{\partial \eta}=\sqrt{\tau^{2}-1} \frac{\partial}{\partial \tau}, & & 1 \leq \tau<\infty, \\
\zeta=\cos \theta, & \frac{\partial}{\partial \theta}=-\sqrt{1-\zeta^{2}} \frac{\partial}{\partial \zeta}, & & -1 \leq \zeta \leq 1 .
\end{array}
$$

The components of the velocity field are expressed in terms of the Stokes stream function $\psi(\tau, \zeta)$ via

$$
\begin{aligned}
& v_{\tau}(\tau, \zeta)=\frac{1}{c^{2} \sqrt{\tau^{2}-\zeta^{2}} \sqrt{\tau^{2}-1}} \frac{\partial \psi(\tau, \zeta)}{\partial \zeta}, \\
& v_{\zeta}(\tau, \zeta)=\frac{1}{c^{2} \sqrt{\tau^{2}-\zeta^{2}} \sqrt{1-\zeta^{2}}} \frac{\partial \psi(\tau, \zeta)}{\partial \tau} .
\end{aligned}
$$

For the vorticity field $\omega$, it is readily verified that

$$
\boldsymbol{\omega}(\tau, \zeta)=\nabla \times \mathbf{v}(\tau, \zeta)=\frac{\hat{\varphi}}{h_{\varphi}} E^{2} \psi(\tau, \zeta),
$$

where

$$
E^{2}=\frac{1}{c^{2}\left(\tau^{2}-\zeta^{2}\right)}\left[\left(\tau^{2}-1\right) \frac{\partial^{2}}{\partial \tau^{2}}+\left(1-\zeta^{2}\right) \frac{\partial^{2}}{\partial \zeta^{2}}\right],
$$

and $h_{\varphi}$ is the relative metric coefficient. By means of Eqs. (3)-(5) all the important fields are obtained once the Stokes stream function $\psi(\tau, \zeta)$ is known. The latter satisfies the equation

$$
E^{4} \psi(\tau, \zeta)=0
$$

For completeness, in solving boundary value problems for Stokes flows associated with spheroidal coordinates, we furnish the Cartesian expression of the outward unit normal vector on the surface of the spheroid $\tau=\tau_{0}$, which is

$$
\hat{\mathbf{n}}\left(\tau_{0}\right)=\frac{1}{\sqrt{\tau_{0}^{2}-\zeta^{2}}}\left(\tau_{0} \sqrt{1-\zeta^{2}} \cos \varphi, \tau_{0} \sqrt{1-\zeta^{2}} \sin \varphi, \sqrt{\tau_{0}^{2}-1 \zeta}\right) .
$$

Note that for any nondegenerate spheroid $S_{\tau_{0}}$, it is $\tau_{0}>1$. Furthermore, $|\zeta| \leq 1$. Hence, the expression $\left(\tau^{2}-\zeta^{2}\right)$ in the denominators of Eqs. (3), (4), (6), and (8) is always bounded away from zero for all points exterior to $S_{\tau_{0}}$, as well as on $S_{\tau_{0}}$.

3. The kernel space of $E^{2}$. Let us represent any function in the kernel of the operator $E^{2}$ in the following separable form

$$
\psi(\tau, \zeta)=T(\tau) Z(\zeta)
$$

Then, it is easily shown that for every $n=0,1,2, \ldots$ the functions $T_{n}(\tau)$ and $Z_{n}(\zeta)$ satisfy the ordinary differential equations

$$
\begin{aligned}
\left(1-\tau^{2}\right) T_{n}^{\prime \prime}(\tau)+n(n-1) T_{n}(\tau) & =0, & & 1<\tau<+\infty \\
\left(1-\zeta^{2}\right) Z_{n}^{\prime \prime}(\zeta)+n(n-1) Z_{n}(\zeta) & =0, & & -1 \leq \zeta \leq 1 .
\end{aligned}
$$


Consequently, both $T_{n}$ and $Z_{n}$ satisfy the same Gegenbauer equation [14] of degree $\left(-\frac{1}{2}\right)$, but over different domains, because $\tau>1$ and $|\zeta| \leq 1$.

The basic formulae related to Gegenbauer functions are furnished in Appendix B. Introducing the eigenfunctions $\boldsymbol{\theta}_{n}^{(i)}$ of the $i$ th kind $(i=1,2,3,4)$ and of order $n(n=0,1,2, \ldots)$ via the formulae

$$
\begin{aligned}
& \Theta_{n}^{(1)}(\tau, \zeta)=G_{n}(\tau) G_{n}(\zeta), \\
& \Theta_{n}^{(2)}(\tau, \zeta)=G_{n}(\tau) H_{n}(\zeta), \\
& \Theta_{n}^{(3)}(\tau, \zeta)=H_{n}(\tau) G_{n}(\zeta), \\
& \Theta_{n}^{(4)}(\tau, \zeta)=H_{n}(\tau) H_{n}(\zeta),
\end{aligned}
$$

the following complete representation of the kernel space of $E^{2}$ is obtained. If $\psi$ belongs to $\operatorname{Ker} E^{2}$, then

$$
\psi(\tau, \zeta)=\sum_{n=0}^{\infty} \sum_{i=1}^{4} A_{n}^{i} \Theta_{n}^{(i)}(\tau, \zeta),
$$

where $A_{n}^{i}$ are constants.

When one is dealing with flows that are regular on the axis of symmetry, all the eigenfunctions of even kind and the leading two eigenfunctions of odd kind should be excluded, as is readily seen from Eqs. (3)-(5). In such cases, the regular-on-theaxis solutions of $E^{2} \psi=0$ are restricted to the proper subspace of $\operatorname{Ker} E^{2}$, which is spanned by the eigenfunctions of odd kind and of order greater than or equal to two. A regular-on-the-axis function, then, has the representation

$$
\psi(\tau, \zeta)=\sum_{n=2}^{\infty} \sum_{i=1,3} A_{n}^{i} \Theta_{n}^{(i)}(\tau, \zeta)
$$

4. Generalized eigenfunctions of $E^{2}$. Every function $\psi$ that belongs to the kernel space of the operator $E^{2}$ is also a solution of Eq. (7). However, these functions do not form a complete set of solutions of Eq. (7). In order to obtain those solutions of Eq. (7) that are not members of the space $\operatorname{Ker} E^{2}$, we use the theory of generalized eigenfunctions. Thus, we seek a complete representation of those functions $\tilde{\psi}(\tau, \zeta)$ for which

$$
E^{2} \tilde{\psi}(\tau, \zeta) \in \operatorname{Ker} E^{2}
$$

In other words, we seek a representation of the preimage, under the map $E^{2}$, of an arbitrary element of the space $\operatorname{Ker} E^{2}$. Obviously, any function $\psi$ that satisfies Eq. (18) automatically solves Eq. (7). Since $E^{2} \tilde{\psi}$ belongs to $\operatorname{Ker} E^{2}$, it has the representation

$$
E^{2} \tilde{\psi}(\tau, \zeta)=\sum_{n=0}^{\infty} \sum_{i=1}^{4} B_{n}^{i} \Theta_{n}^{(i)}(\tau, \zeta)
$$

as it is dictated by Eq. (16). 
Let us assume that $\Omega_{n}^{(i)}(\tau, \zeta)$ denotes the preimage of the eigenfunction $\Theta_{n}^{(i)}(\tau, \zeta)$ under the operator $E^{2}$, in other words, that $\Omega_{n}^{(i)}(\tau, \zeta)$ are solutions of the equations

$$
c^{2} E^{2} \Omega_{n}^{(i)}(\tau, \zeta)=\Theta_{n}^{(i)}(\tau, \zeta)
$$

for each $i=1,2,3,4$ and $n=0,1,2, \ldots$. The constant $c^{2}$ enters Eq. (20) for notational convenience. The functions $\Omega_{n}^{(i)}$ are the generalized eigenfunctions of $E^{2}$ that correspond to the zeroth eigenvalue. By virtue of the linearity of the operator $E^{2}$, if $\tilde{\psi}$ is a solution of relation (18) that does not belong to $\operatorname{Ker} E^{2}$, and if it assumes the representation

$$
\tilde{\psi}(\tau, \zeta)=\sum_{n=0}^{\infty} \sum_{i=1}^{4} B_{n}^{i} \Omega_{n}^{(i)}(\tau, \zeta)
$$

in terms of the generalized eigenfunctions $\Omega_{n}^{(i)}$, then Eq. (20) implies that

$$
\begin{aligned}
c^{2} E^{2} \tilde{\psi}(\tau, \zeta) & =\sum_{n=0}^{\infty} \sum_{i=1}^{4} B_{n}^{i} c^{2} E^{2} \Omega_{n}^{(i)}(\tau, \zeta) \\
& =\sum_{n=0}^{\infty} \sum_{i=1}^{4} B_{n}^{i} \Theta_{n}^{i}(\tau, \zeta) .
\end{aligned}
$$

Consequently, Eq. (20) effects a complete spectral decomposition of the solution space $\operatorname{Ker} E^{4}$, once the generalized eigenfunctions $\Omega_{n}^{(i)}$ are obtained.

Below, we solve the partial differential equations (20) for every $i=1,2,3,4$ and every $n=0,1,2, \ldots$. The peculiarities of the first two Gegenbauer functions make it necessary to treat the cases $n=0,1,2$ and $n=3$ separately. The cases $n \geq 4$ can be investigated collectively.

A particular solution of the inhomogeneous linear partial differential equation

$$
\left[\left(\tau^{2}-1\right) \partial_{\tau \tau}+\left(1-\zeta^{2}\right) \partial_{\zeta \zeta}\right] \psi(\tau, \zeta)=f_{n}(\tau) g_{m}(\zeta),
$$

where $f_{n}$ and $g_{m}$ are Gegenbauer functions of the first or second kind and of order $n$ and $m$, respectively, with $n \neq m$ and $n+m \neq 1$, is provided by the separable function

$$
\psi_{n m}(\tau, \zeta)=\frac{f_{n}(\tau) g_{m}(\zeta)}{(n-m)(n+m-1)}
$$

for $n \geq 0, m \geq 0$. The proof follows easily from Gegenbauer equations (10) and (11). The factor $\left(\tau^{2}-\zeta^{2}\right)$ in relation (6) ensures that all products on the right-hand sides of the differential equations (23) involve polynomials of different order, hence, the $n-m$ factor in (24) causes no problem in the way the particular solutions are obtained.

In order to solve Eq. (20) for a particular combination of $i$ and $n$ we write it in the form

$$
\left[\left(\tau^{2}-1\right) \partial_{\tau \tau}+\left(1-\zeta^{2}\right) \partial_{\zeta \zeta}\right] \Omega_{n}^{(i)}(\tau, \zeta)=\left(\tau^{2}-\zeta^{2}\right) \Theta_{n}^{(i)}(\tau, \zeta)
$$

and use relations (B.14)-(B.23) to absorb the $\tau^{2}$ and the $\zeta^{2}$ factors into Gegenbauer functions of translated order. Then Eq. (24) is used to write down the appropriate 
particular solutions. This procedure has to be carried out independently for $n=$ $0,1,2,3$ and, in general, for every $n \geq 4$ and, of course, for each $i=1,2,3,4$.

As an illustration we show how the generalized eigenfunction $\Omega_{2}^{(2)}(\tau, \zeta)$ is obtained. For $i=n=2$ Eq. (25) becomes

$$
\left[\left(\tau^{2}-1\right) \partial_{\tau \tau}+\left(1-\zeta^{2}\right) \partial_{\zeta \zeta}\right] \Omega_{2}^{(2)}(\tau, \zeta)=\left(\tau^{2}-\zeta^{2}\right) G_{2}(\tau) H_{2}(\zeta),
$$

where the expression for $\Theta_{2}^{(2)}(\tau, \zeta)$ is taken from Eq. (13). From (B.16) and (B.21) we now conclude that

$$
\begin{aligned}
& {\left[\left(\tau^{2}-1\right) \partial_{\tau \tau}+\left(1-\zeta^{2}\right) \partial_{\zeta \zeta}\right] \Omega_{2}^{(2)}(\tau, \zeta)} \\
& \quad=\frac{1}{3} G_{2}(\tau) G_{1}(\zeta)+\frac{4}{5} G_{4}(\tau) H_{2}(\zeta)-\frac{4}{5} G_{2}(\tau) H_{4}(\zeta) .
\end{aligned}
$$

Finally, the linearity of Eq. (27) permits the term-by-term use of Eq. (24) and therefore the evaluation of the particular solution

$$
\Omega_{2}^{(2)}(\tau, \zeta)=\frac{2}{25}\left[G_{2}(\tau) H_{4}(\zeta)+G_{4}(\tau) H_{2}(\zeta)\right]+\frac{1}{6} G_{2}(\tau) G_{1}(\zeta)
$$

Note that all we need from Eq. (25) is just a particular solution, since the general solution of the corresponding homogeneous equation is a function that belongs to the space $\operatorname{Ker} E^{2}$, for which we already have a complete representation.

After some tedious calculations, along the lines of the above example, we have obtained all the generalized eigenfunctions of $E^{2}$ which are given in Appendix C.

Once the generalized eigenfunctions are obtained, any Stokes stream function $\psi$ can be represented as

$$
\psi(\tau, \zeta)=\sum_{n=0}^{\infty} \sum_{i=1}^{4}\left[A_{n}^{i} \Theta_{n}^{(i)}(\tau, \zeta)+B_{n}^{i} \Omega_{n}^{(i)}(\tau, \zeta)\right],
$$

where the sum with the $A$-coefficients represents an element of the space $\operatorname{Ker} E^{2}$, whereas the sum with the $B$-coefficients stands for a function that is mapped to the space $\operatorname{Ker} E^{2}$ under the action of the operator $E^{2}$.

In view of the formulae (3)-(5), it is easily seen that the demand for solutions having velocity and vorticity fields that are regular on the axis restricts the representation to

$$
\psi(\tau, \zeta)=\sum_{n=2}^{\infty} \sum_{i=1,3}\left[A_{n}^{i} \Theta_{n}^{(i)}(\tau, \zeta)+B_{n}^{i} \Omega_{n}^{(i)}(\tau, \zeta)\right] .
$$

Expansion (30) represents the solution of the most usual axisymmetric Stokes flow problems in spheroidal coordinates.

Each eigenfunction $\Theta_{n}^{(i)}$ and each generalized eigenfunction $\Omega_{n}^{(i)}$ corresponds to a basic Stokes flow field. Equation (29) expresses the fact that any axisymmetric Stokes flow can be written as an appropriate linear combination of these basic flow fields. Streamlines of the flow fields represented by $\Omega_{0}^{(1)}, \Omega_{0}^{(2)}, \Omega_{0}^{(3)}, \Omega_{0}^{(4)}, \Omega_{2}^{(1)}$, $\Omega_{2}^{(2)}, \Omega_{2}^{(3)}$, and $\Omega_{2}^{(4)}$ are given in Figs. 1 to 8 (see pp. 164-167). Streamlines for $\Omega_{1}^{(1)}, \Omega_{1}^{(2)}, \Omega_{1}^{(3)}, \Omega_{1}^{(4)}$ are not given separately, in view of Eqs. (C.5)-(C.8). 


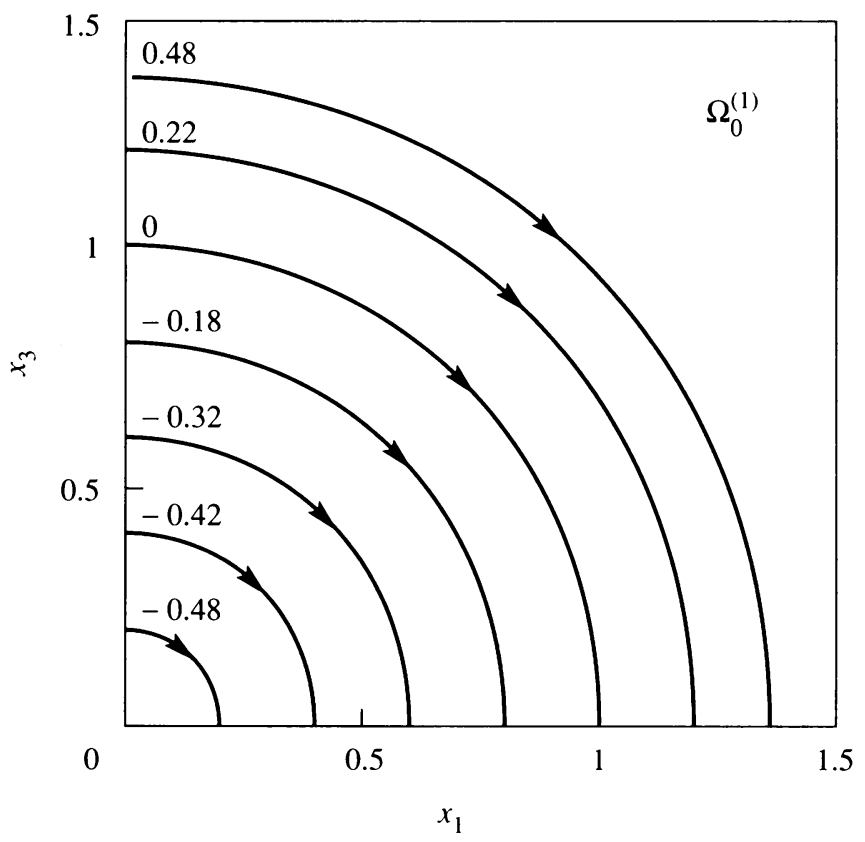

Fig. 1. Streamlines of the basic flow field represented by the generalized eigenfunction $\Omega_{0}^{(1)}$ for $c=1$. Note also Eq. (C.8).

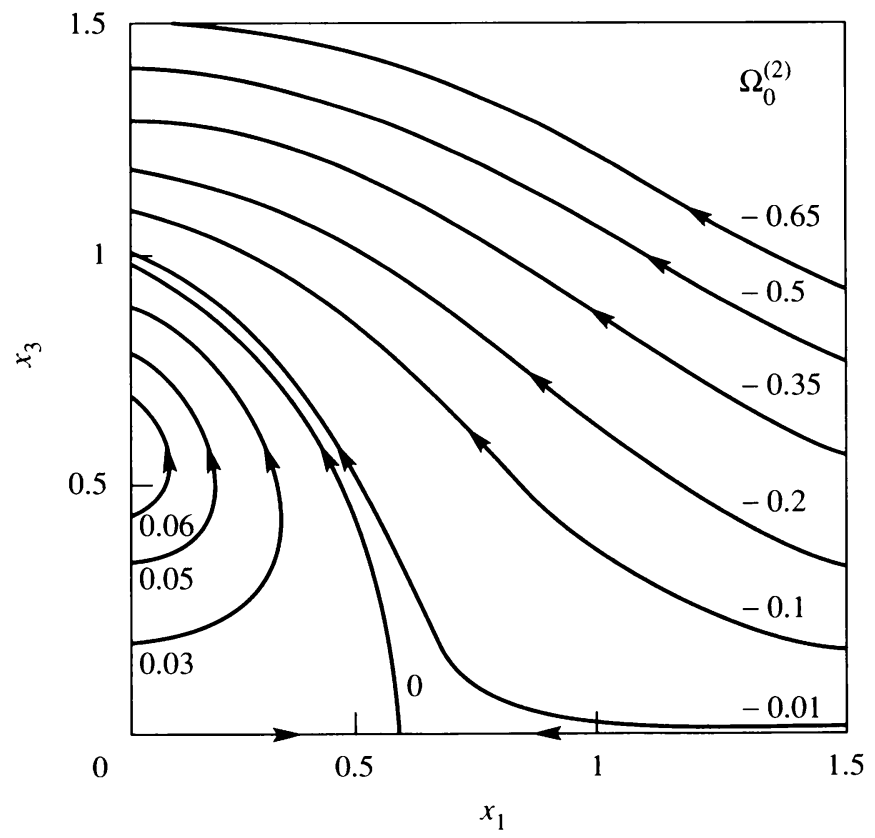

Fig. 2. Streamlines of the basic flow field represented by the generalized eigenfunction $\Omega_{0}^{(2)}$ for $c=1$. Note also Eq. (C.7). 


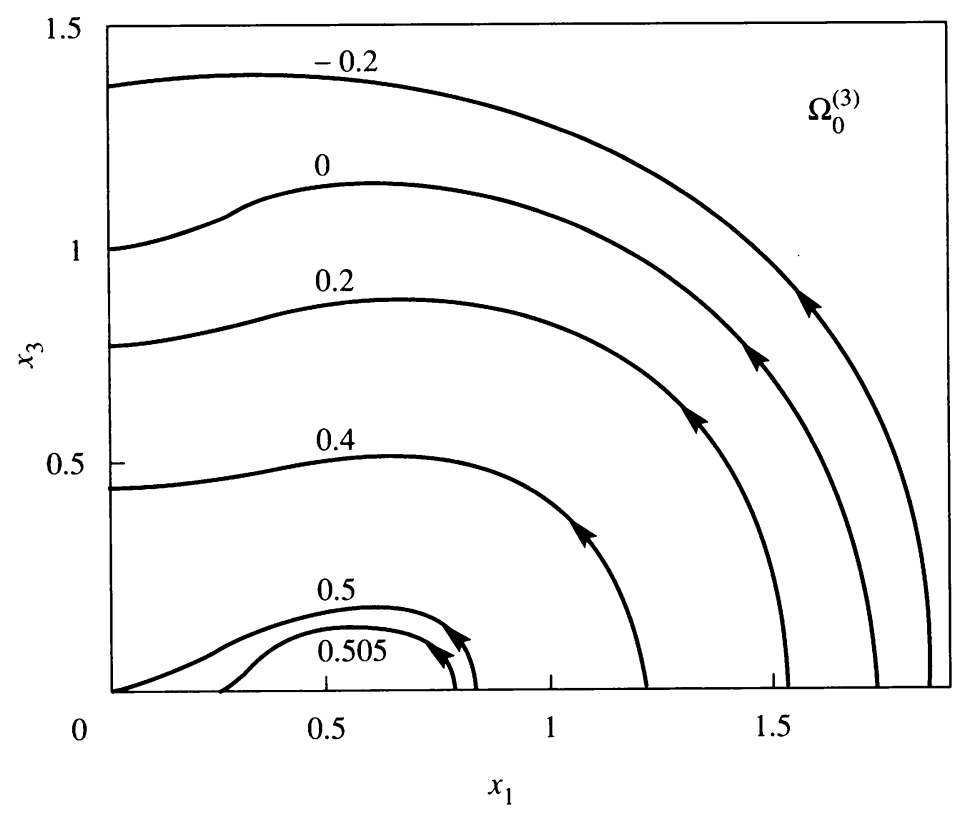

Fig. 3. Streamlines of the basic flow field represented by the generalized eigenfunction $\Omega_{0}^{(3)}$ for $c=1$. Note also Eq. (C.6).

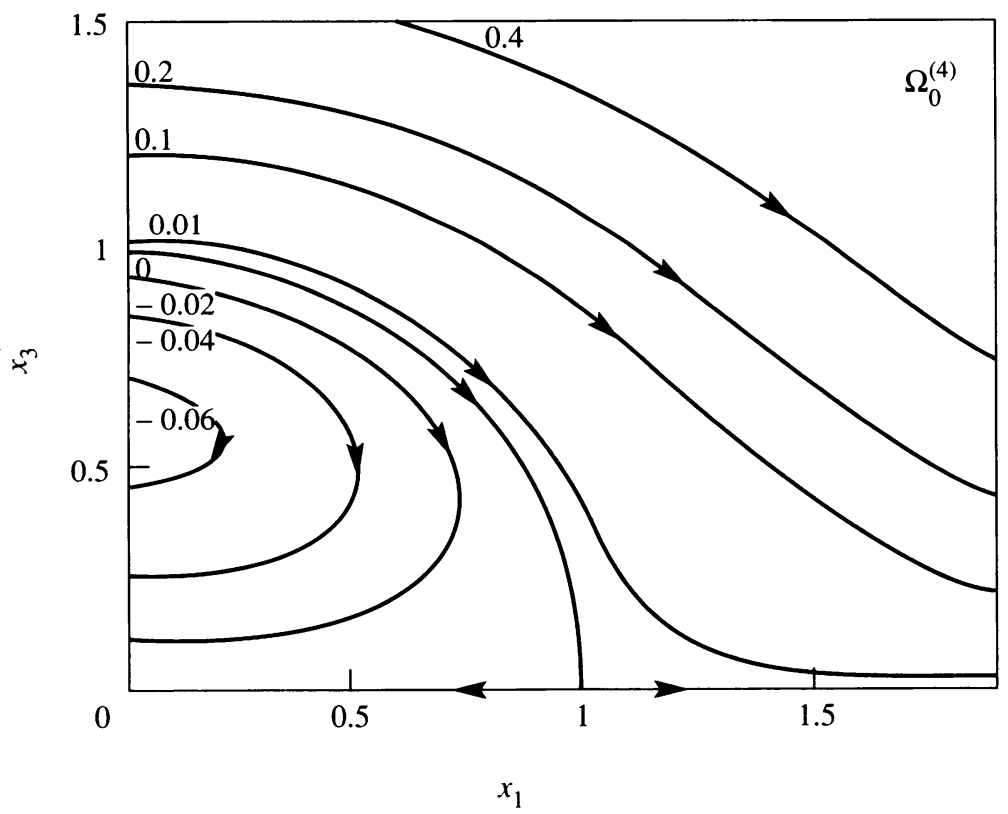

Fig. 4. Streamlines of the basic flow field represented by the generalized eigenfunction $\Omega_{0}^{(4)}$ for $c=1$. Note also Eq. (C.5). 


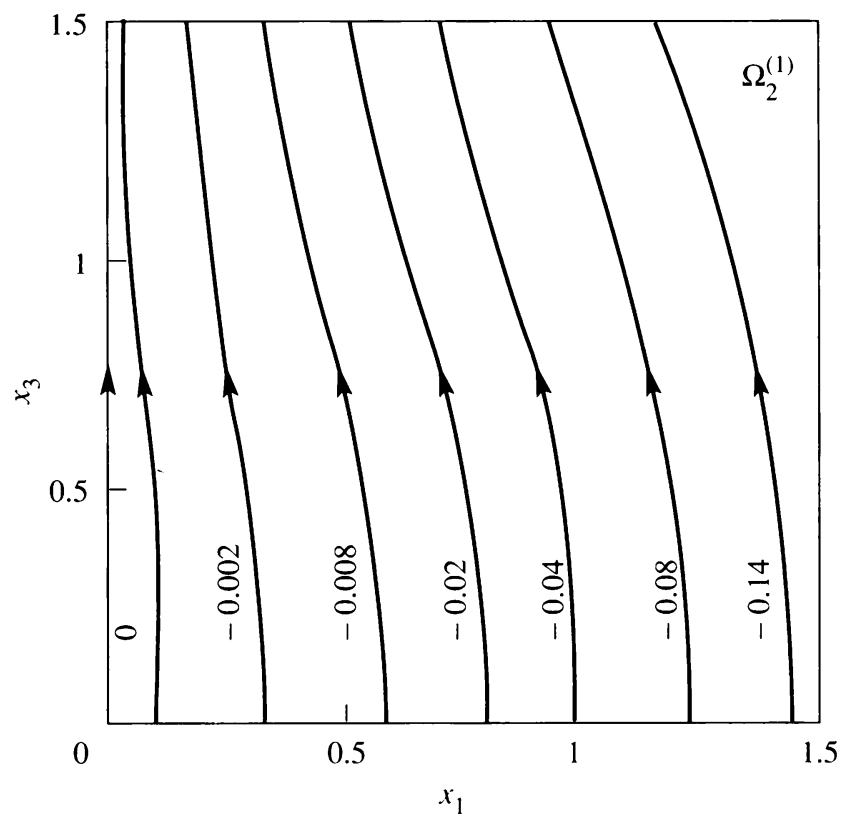

Fig. 5. Streamlines of the basic flow field represented by the generalized eigenfunction $\Omega_{2}^{(1)}$ for $c=1$.

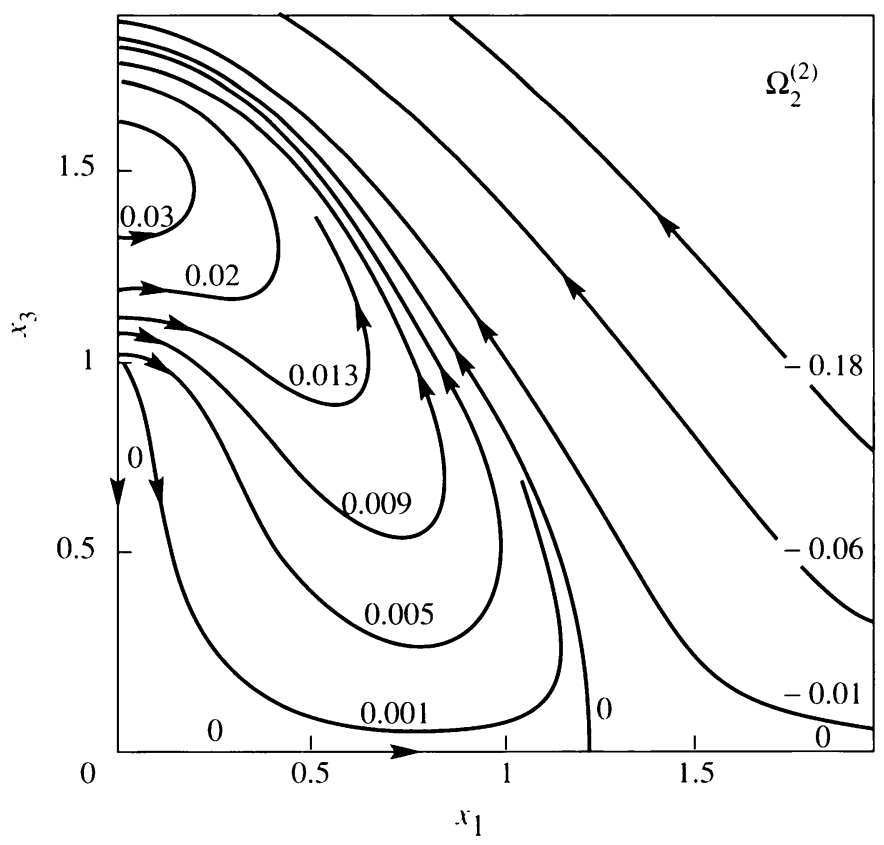

Fig. 6. Streamlines of the basic flow field represented by the generalized eigenfunction $\Omega_{2}^{(2)}$ for $c=1$. 


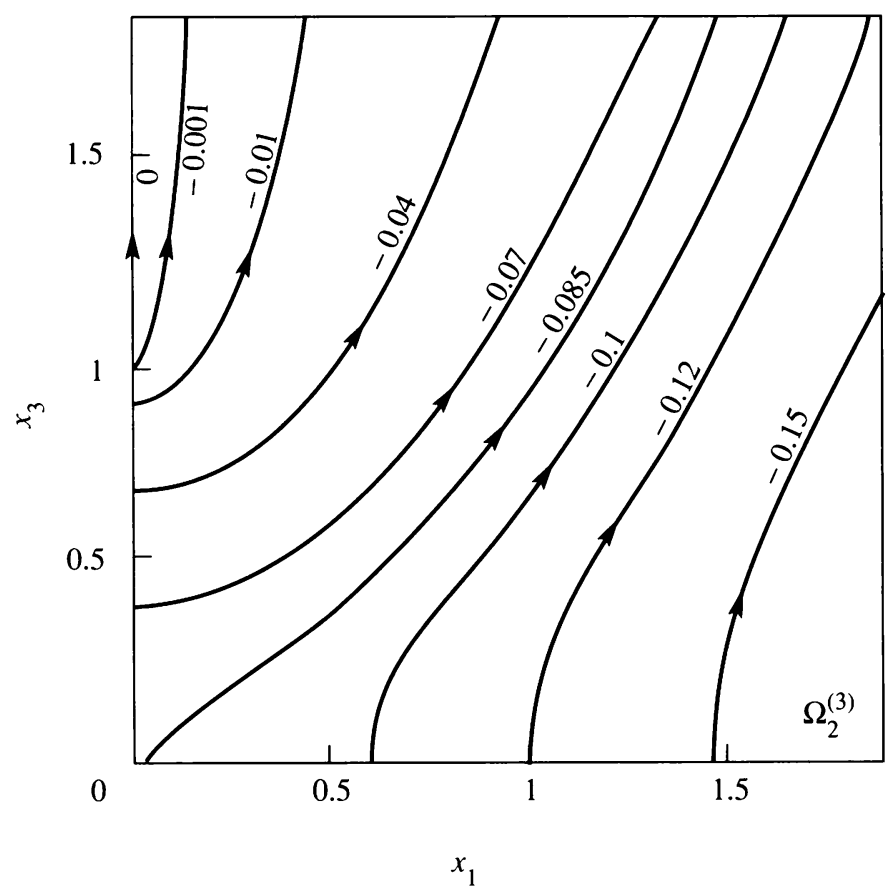

Fig. 7. Streamlines of the basic flow field represented by the generalized eigenfunction $\Omega_{2}^{(3)}$ for $c=1$.

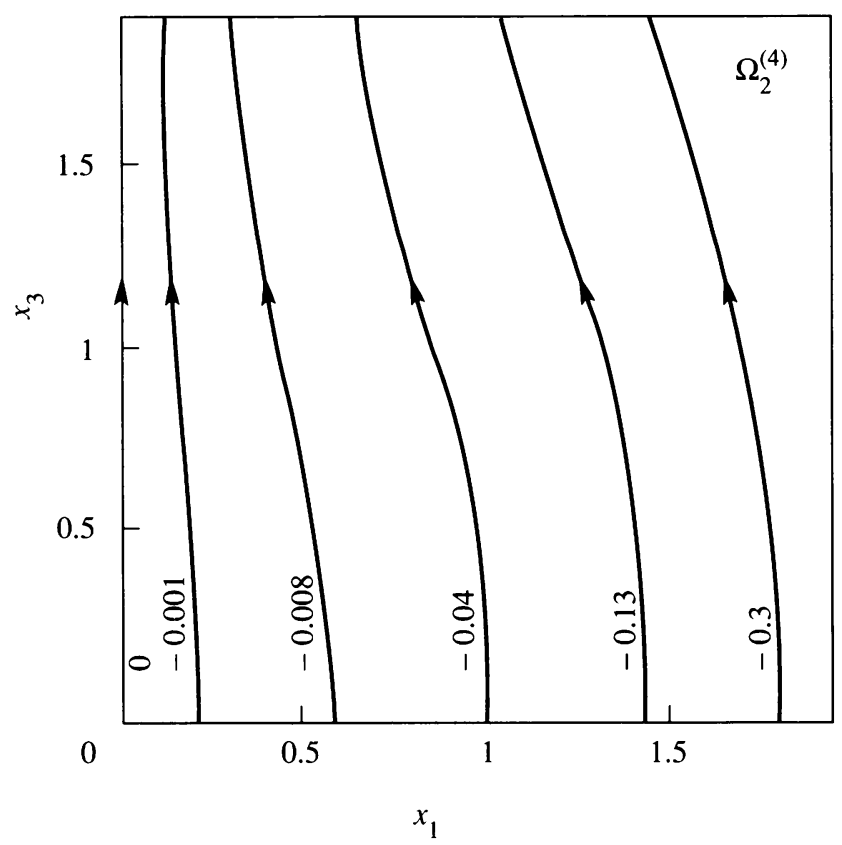

Fig. 8. Streamlines of the basic flow field represented by the generalized eigenfunction $\Omega_{2}^{(4)}$ for $c=1$. 
5. Semiseparable solutions. The main characteristic of the simple and generalized eigenfunction expansion (29) is that, although every term of the series solves Eq. (7), the $\zeta$-dependence does not enter through the Gegenbauer functions of fixed order. Instead, every term of the expansion (29) involves a finite number of Gegenbauer functions of mixed order.

In the interest of solving standard boundary value problems, with or without singularities along the axis of symmetry, an expansion of the general stream function in terms of Gegenbauer functions for the $\zeta$-dependence is needed. This is obtained by rearranging the terms of Eq. (29) and collecting all of the $\tau$-dependent terms associated with each $\zeta$-dependent Gegenbauer function. This procedure leads to the expansion

$$
\psi(\tau, \zeta)=g_{0}(\tau) G_{0}(\zeta)+g_{1}(\tau) G_{1}(\zeta)+\sum_{n=2}^{\infty}\left[g_{n}(\tau) G_{n}(\zeta)+h_{n}(\tau) H_{n}(\zeta)\right]
$$

The $\tau$-dependent functions $g_{n}(\tau)$ and $h_{n}(\tau)$ are given, for convenience, in Appendix D.

It is interesting to note that the individual terms of the expansion (31) are not solutions of Eq. (7), but the full expansion is. The complicated expressions, through which the $A$ 's and the $B$ 's enter the $\tau$-functions $g_{n}$ and $h_{n}$, Appendix D, reflect the appropriate way in which the individual terms should interweave in order for the expansion (31) to form a solution of Eq. (7).

We observe that the functions $g_{n}(\tau)$ are always associated with the $G_{n}(\zeta)$ polynomials, whereas the functions $h_{n}(\tau)$ are always associated with the $H_{n}(\zeta)$ functions. Since $g_{n}(\tau)$ and $h_{n}(\tau)$ do not coincide, it follows that Eq. (7) does not accept genuinely separable solutions. Nevertheless, expansion (31) exhibits some kind of separation, which we will refer to as semiseparation to indicate that the solution can be written as a sum of particular products of functions with $\tau$ and $\zeta$ dependence alone but not in any combination.

Actually, the operator $E^{2}$, as it is given by (6), is the product of an algebraic and a differential factor. The elements of the space $\operatorname{ker} E^{2}$ accept a separable form, since the algebraic factor of $E^{2}$ is eliminated from the equation $E^{2} \psi=0$. On the other hand, it is this algebraic factor of $E^{2}$ that destroys the separability of Eq. (7) through the composition $E^{2} \circ E^{2}$. Therefore, it seems that the loss of separability is not of a "very serious" nature and indeed it is not since the generalized solutions are not constructed out of "new" functions. The generalized solutions are formed by following a particular weaving pattern with the indices of the same Gegenbauer functions that were used to construct the separable solution. This is exactly what we characterize as semiseparation.

For solutions that are regular on the axis, the representations (B.10) and (B.11) can be used to write the general solution in terms of the orthogonal Gegenbauer polynomials $\left\{G_{n}(\zeta), n \geq 2\right\}$ as

$$
\begin{aligned}
\psi(\tau, \zeta)= & \sum_{n=1}^{\infty}\left[(4 n-1) g_{0}(\tau)+g_{2 n}(\tau)\right] G_{2 n}(\zeta) \\
& +\sum_{n=1}^{\infty}\left[-(4 n+1) g_{1}(\tau)+g_{2 n+1}(\tau)\right] G_{2 n+1}(\zeta)
\end{aligned}
$$


The representation (32) for the general, regular-on-the-axis, Stokes stream function is very useful for particular applications because of the orthogonality relations (B.13).

6. Reduction to the sphere. The spheroidal coordinate system (A.9) reduces to the spherical coordinate system in the limit, as the semifocal distance $c$ tends to zero. From (A.9) it is readily obtained that

$$
r=c \sqrt{\tau^{2}+\zeta^{2}-1}
$$

which, in view of (A.10), provides the limit

$$
\lim _{c \rightarrow 0+} c \tau=r
$$

Consequently, as the prolate spheroid (A.11) deforms continuously by bringing its foci close together, the radial spherical coordinate is recovered from the limit of the product $c \tau$, as $c \rightarrow 0+$, while the form of the angular dependence remains intact. This implies that the Gegenbauer functions still provide the angular dependence, whereas the radial dependence is formally obtained from the corresponding Gegenbauer functions as follows: first replace $\tau$ by $\tau / c$, then take the limit of $c^{n} G_{n}(\tau / c)$ and $c^{1-n} H_{n}(\tau / c)$ as $c \rightarrow 0+$, and replace $\tau$ by $r$.

The expansion

$$
\frac{1}{2} \ln \frac{\tau+1}{\tau-1}=\operatorname{coth}^{-1} \tau=\frac{1}{\tau}+\frac{1}{3 \tau^{3}}+\frac{1}{5 \tau^{5}}+\cdots
$$

for $\tau>1$ leads to the limit

$$
\lim _{c \rightarrow 0+} \frac{1}{2 c} \ln \frac{\tau+1}{\tau-1}=\frac{1}{r}
$$

The operator $E^{2}$, given by Eq. (6), in the limit as $c \rightarrow 0+$, provides

$$
E^{2}=\frac{\partial^{2}}{\partial r^{2}}+\frac{\sin \theta}{r^{2}} \frac{\partial}{\partial \theta}\left(\frac{1}{\sin \theta} \frac{\partial}{\partial \theta}\right)
$$

which is the form that $E^{2}$ assumes in spherical coordinates.

The Gegenbauer functions transform as follows, in the limit as $c \rightarrow 0+$,

$$
\left.\begin{array}{c}
c^{0} G_{0}(\tau) \rightarrow 1, \quad c G_{1}(\tau) \rightarrow-r, \quad c^{2} G_{2}(\tau) \rightarrow-\frac{1}{2} r^{2}, \\
c^{3} G_{3}(\tau) \rightarrow-\frac{1}{2} r^{3}, \quad c^{4} G_{4}(\tau) \rightarrow-\frac{5}{8} r^{4} \\
c^{n} G_{n}(\tau) \rightarrow(\text { const }) r^{n}, \quad n \geq 5 ;
\end{array}\right\}
$$


Therefore, in order to recover the spherical form of the Stokes stream function from the corresponding spheroidal expressions, we need to introduce the following scaling for the constants:

(i) $A_{0}^{1}, A_{0}^{2}, A_{1}^{3}, A_{1}^{4}$ remain the same;

(ii) $A_{1}^{1}, A_{1}^{2}, A_{0}^{3}, A_{0}^{4}$ are multiplied by $c$;

(iii) for $n \geq 2, A_{n}^{1}, A_{n}^{2}$ are multiplied by $c^{n}$;

(iv) for $n \geq 2, A_{n}^{3}, A_{n}^{4}$ are multiplied by $c^{1-n}$;

and by virtue of (20), which introduces an extra factor of $c^{2}$ :

(v) $B_{0}^{1}, B_{0}^{2}, B_{1}^{3}, B_{1}^{4}$ are multiplied by $c^{2}$;

(vi) $B_{1}^{1}, B_{1}^{2}, B_{0}^{3}, B_{0}^{4}$ are multiplied by $c^{3}$;

(vii) for $n \geq 2, B_{n}^{1}, B_{n}^{2}$ are multiplied by $c^{n+2}$;

(viii) for $n \geq 2, B_{n}^{3}, B_{n}^{4}$ are multiplied by $c^{3-n}$.

Introducing the above scaling and sending $c \rightarrow 0+$ into the expressions for the $g$ 's and the $h$ 's we obtain the following combinations of terms:

$$
g_{n}(\tau) \underset{c \rightarrow 0+}{\rightarrow} \Gamma_{n}^{1} r^{n}+\Gamma_{n}^{2} \frac{1}{r^{n-1}}+\Gamma_{n}^{3} r^{n+2}+\Gamma_{n}^{4} \frac{1}{r^{n-3}}, \quad n \geq 0
$$

and

$$
h_{n}(\tau) \underset{c \rightarrow 0+}{\rightarrow} \Delta_{n}^{1} r^{n}+\Delta_{n}^{2} \frac{1}{r^{n-1}}+\Delta_{n}^{3} r^{n+2}+\Delta_{n}^{4} \frac{1}{r^{n-3}}, \quad n \geq 2,
$$

where $\Gamma_{n}^{i}, \Delta_{n}^{i}$ are constants.

If we substitute Eqs. (38) and (39) into the general expansion (31), we arrive at the well-known representation of a stream function in spherical coordinates (see, e.g., [9]).

The same general expansion can be derived if the above scaling is applied to the expansion (16) of an element in the space $\operatorname{Ker} E^{2}$, as well as to Eq. (20). Then Eq. (20) is solved with $E^{2}$ given by Eq. (37).

7. Application. In order to demonstrate the usefulness of the present method, we use it to solve Stokes flow within a fluid cell contained between two confocal spheroidal surfaces. The internal spheroidal surface $S_{\alpha}$ is solid, whereas the external one $S_{\beta}$ is the ficticious boundary of a unit cell (spheroid-in-cell) that can be used to model flow through a swarm of spheroidal particles. The volume of the fluid cell is chosen so that the solid volume fraction in the cell equals the solid volume fraction of the swarm.

For the purposes of the present example, we apply boundary conditions which are analogous to those of the Kuwabara's [11] sphere-in-cell model. [Application of boundary conditions analogous to those of Happel's [8] sphere-in-cell model lead to a somewhat more complicated analysis because of the fourth boundary condition, as can be seen in a forthcoming publication. We also restrict our attention to prolate spheroids, since the solution for oblate spheroids can be obtained through a simple transformation.]

In what follows, all lengths have been rendered dimensionless using the short semiaxis of the internal spheroid, $\tilde{a}_{1}$, as characteristic length. The stream function is 
rendered dimensionless through division by the characteristic value $\tilde{u} \tilde{a}_{1}^{2}$, where $\tilde{u}$ is the approach velocity.

Let $a_{1}(=1)$ and $a_{3}$ be the short and long (dimensionless) semiaxes of the internal spheroid, respectively, and $b_{1}$ and $b_{3}$ those of the external one. The two spheroids are confocal with semifocal length $c$. The values of $\tau$ on the internal and external spheroid are

$$
\begin{gathered}
\tau_{\alpha}=\cosh \alpha=\frac{a_{3}}{c}, \\
\tau_{\beta}=\cosh \beta=\frac{b_{3}}{c},
\end{gathered}
$$

respectively.

We assume that the solid spheroid is stationary and that an axisymmetric flow is caused in the fluid layer $\left(\tau_{\alpha} \leq \tau \leq \tau_{\beta}\right)$ by a uniform approach velocity on the external spheroid. This approach velocity is assumed to have magnitude $\tilde{u}$ and to be directed in the positive direction of the $x_{3}$-axis. Futhermore, the vorticity is assumed to become nil on the external spheroid.

Assuming Stokes flow conditions, the equation of motion is given by Eq. (7). The boundary conditions can be expressed as

$$
\begin{array}{ll}
\text { B.C.1. } & \psi=0 \quad \text { on } \tau=\tau_{\alpha} \\
\text { B.C.2. } & \frac{\partial \psi}{\partial \tau}=0 \quad \text { on } \tau=\tau_{\alpha}, \\
\text { B.C.3. } & \frac{\partial \psi}{\partial \zeta}=-c^{2}\left(\tau^{2}-1\right) \zeta \quad \text { on } \tau=\tau_{\beta}, \\
\text { B.C.4. } & {\left[\left(\tau^{2}-1\right) \frac{\partial^{2}}{\partial \tau^{2}}+\left(1-\zeta^{2}\right) \frac{\partial^{2}}{\partial \zeta^{2}}\right] \psi=0 \quad \text { on } \tau=\tau_{\beta} .}
\end{array}
$$

Equation (7) is to be integrated with the B.C.s given by Eqs. (43)-(46). As a consequence of the semiseparability, it is actually not possible to satisfy exactly the equation and the given boundary conditions at the same time. If we consider the semiseparable form (31) of the solution then we have an infinite number of approximations for the solution. In order to obtain a unique solution, it will be necessary to impose an additional limit condition of geometrical nature, namely, that the solution sought tends asymptotically to the solution for the spherical case (the Kuwabara solution) as the prolate spheroid tends to become spherical $\left(c \rightarrow 0+, a_{3} \rightarrow 1\right)$. Actually, this geometric condition is equivalent to the assumption of separability imposed by Happel and Brenner [9]. Both conditions restrict the solution space as much as is needed in order for the problem to secure a unique solution.

Now, the Gegenbauer functions have the following characteristics: $G_{n}(\zeta)$ are regular on the $x_{3}$-axis; $H_{n}(\zeta)$ are singular on the $x_{3}$-axis; $G_{n}(\tau)$ are regular in the interior of the spheroid $S_{\alpha}$; and $H_{n}(\tau)$ are regular everywhere (since $\tau>1$ ), except on the focal segment (where $\tau=1$ ). As we demand of our solution to be regular 
on the $x_{3}$-axis and in the space between $S_{\alpha}$ and $S_{\beta}$, we use for the representation of our solution only the terms $g_{n}(\tau) G_{n}(\zeta)$. Furthermore, taking into account the symmetry of the $\psi$-field on either side of the equatorial plane $(\zeta=0)$, we retain only the even terms of the solution. Thus we obtain

$$
\psi(\tau, \zeta)=\sum_{n=2,4, \ldots}^{\infty} g_{n}(\tau) G_{n}(\zeta)
$$

where the sum runs through the even integers. Now, using the expressions (D.3) and (D.5) for $g_{n}(\tau)$ we obtain

$$
\begin{aligned}
\psi(\tau, \zeta)= & \frac{c^{2} B_{2}}{6} G_{1}(\tau) G_{2}(\zeta)+\sum_{n=2,4, \ldots}^{\infty}\left[\mathscr{A}_{n+2} G_{n+2}(\tau)+\mathscr{B}_{n+2} H_{n+2}(\tau)\right] G_{n}(\zeta) \\
& +\sum_{n=2,4, \ldots}^{\infty}\left[\mathscr{A}_{n+2} G_{n}(\tau)+\mathscr{B}_{n+2} H_{n}(\tau)\right] G_{n+2}(\zeta) \\
& +\sum_{n=2,4, \ldots}^{\infty}\left[C_{n} G_{n}(\tau)+D_{n} H_{n}(\tau)\right] G_{n}(\zeta)
\end{aligned}
$$

where

$$
\begin{aligned}
& \mathscr{A}_{n+2}=\frac{c^{2}}{2(2 n+1)}\left[A_{n} \beta_{n}-A_{n+2} \alpha_{n+2}\right], \\
& \mathscr{B}_{n+2}=\frac{c^{2}}{2(2 n+1)}\left[B_{n} \beta_{n}-B_{n+2} \alpha_{n+2}\right] .
\end{aligned}
$$

Here $A_{n}, B_{n}, C_{n}$, and $D_{n}$ are constants to be determined from the appropriate B.C.s, whereas the coefficients $\alpha_{n}$ and $\beta_{n}$ are given by (B.24) and (B.25), respectively.

Using the relations

$$
G_{n}^{\prime}(\zeta)=-P_{n-1}(\zeta), \quad G_{n}(\zeta)=\frac{P_{n-2}(\zeta)-P_{n}(\zeta)}{2 n-1}, \quad n=2,3,
$$

and taking into account that

$$
\zeta=-G_{1}(\zeta)=\sum_{n=1}^{\infty}(4 n+1) G_{2 n+1}(\zeta)
$$

[note that $G_{1}(\zeta)$ is not orthogonal to the other members of the family, $G_{n}(\zeta)$, $n \geq 2$ ], the B.C.s can be written as follows:

$$
\begin{array}{ll}
\text { B.C.(1) } & g_{2}\left(\tau_{\alpha}\right)=0, \\
\text { B.C.(2) } & g_{2}^{\prime}\left(\tau_{\alpha}\right)=0, \\
\text { B.C.(3) } & g_{2}\left(\tau_{\beta}\right)=c^{2}\left(\tau_{\beta}^{2}-1\right), \\
\text { B.C.(4) } & \left(1-\tau_{\beta}^{2}\right) g_{2}^{\prime \prime}\left(\tau_{\beta}\right)+2 g_{2}\left(\tau_{\beta}\right)=0 \quad\left[\text { or } g_{2}^{\prime \prime}\left(\tau_{\beta}\right)=2 c^{2}\right]
\end{array}
$$


and for $n=4,6,8, \ldots$

$$
\begin{array}{ll}
\text { B.C.(5) } & g_{n}\left(\tau_{\alpha}\right)=0, \\
\text { B.C.(6) } & g_{n}^{\prime}\left(\tau_{\alpha}\right)=0, \\
\text { B.C.(7) } & g_{n}\left(\tau_{\beta}\right)=0, \\
\text { B.C.(8) } \left.\left(1-\tau_{\beta}^{2}\right) g_{n}^{\prime \prime}\left(\tau_{\beta}\right)+n(n-1) g_{n}\left(\tau_{\beta}\right)=0 \quad \text { [or } g_{n}^{\prime \prime}\left(\tau_{\beta}\right)=0\right] \text {. }
\end{array}
$$

We observe that B.C.(1)-(4) are not sufficient to determine the five constants (for $n=2$ ) in Eq. (48). Therefore, the stream function for this particular problem, in the system of spheroidal coordinates, is not uniquely determined by the standard B.C.s. Instead, we have a "rich" space of approximate solutions, and we need to provide an additional condition in order to determine a unique solution.

Here, we complete the formulation for the problem by imposing the following additional condition. As $c \rightarrow 0+$, we have $\alpha \rightarrow \infty$ and $\tau_{\alpha} \rightarrow \infty$ (as well as $\left.\beta \rightarrow \infty, \tau_{\beta} \rightarrow \infty\right)$ and both spheroidal surfaces tend to become spherical. Hence, in the limit as $c \rightarrow 0+$, the solution, Eq. (48), should tend to the Kuwabara solution, which in spherical coordinates has the form

$$
\psi_{K}(r, \theta)=\left(A r+B r^{2}+C r^{4}+D r^{-1}\right) \sin ^{2} \theta
$$

In order to proceed we need to determine the behavior of the solution sought in the limit as $c \rightarrow 0+$, following the rules developed in the previous section. The following scaling is required to ensure that all Gegenbauer functions go to the corresponding powers of $r$, as $c \rightarrow 0+$ :

$$
\begin{array}{ll}
\bar{A}_{n}=c^{-n} A_{n}, & \bar{B}_{n}=c^{n-1} B_{n}, \\
\bar{C}_{n}=c^{-n} C_{n}, & \bar{D}_{n}=c^{n-1} D_{n} .
\end{array}
$$

Now, (48) can be written in the form

$$
\begin{aligned}
\psi(\tau, \zeta)= & \sum_{n=2,4, \ldots}^{\infty} \Gamma_{n, 0}(\tau) G_{n}(\zeta)+c^{2} \sum_{n=2,4, \ldots}^{\infty} \Gamma_{n, 2}(\tau) G_{n}(\zeta) \\
& +c^{4} \sum_{n=2,4, \ldots}^{\infty} \Gamma_{n, 4}(\tau) G_{n}(\zeta)
\end{aligned}
$$

where $\Gamma_{i, j}(\tau)$ are functions of $\tau$ that reduce to sums of powers of $r$, as $c \rightarrow 0+$. 
Specifically,

$$
\begin{aligned}
& \Gamma_{2,0}(\tau)= \frac{\bar{B}_{2}}{6} c G_{1}(\tau)+\frac{2 \bar{A}_{2}}{25} c^{4} G_{4}(\tau)+\bar{C}_{2} c^{2} G_{2}(\tau)+\bar{D}_{2} \frac{H_{2}(\tau)}{c} \\
& \Gamma_{4,0}(\tau)= \frac{-\bar{B}_{4}}{175} \frac{H_{2}(\tau)}{c}+\frac{\bar{A}_{4}}{189} c^{6} G_{6}(\tau)+\bar{C}_{4} c^{4} G_{4}(\tau)+\bar{D}_{4} \frac{H_{4}(\tau)}{c^{3}} \\
& \Gamma_{n, 0}(\tau)= \frac{-(n-3)(n-2)}{2(2 n-1)(2 n-3)^{2}} \bar{B}_{n} \frac{H_{n-2}}{c^{n-3}}+\frac{(n+1)(n+2)}{2(2 n-1)(2 n+1)^{2}} \bar{A}_{n} c^{n+2} G_{n+2}(\tau) \\
&+\bar{C}_{n} c^{n} G_{n}(\tau)+\bar{D}_{n} \frac{H_{n}(\tau)}{c^{n-1}} \\
& \Gamma_{2,2}(\tau)=-\frac{\bar{B}_{4}}{175} \frac{H_{4}(\tau)}{c^{3}}-\frac{\bar{A}_{4}}{175} c^{4} G_{4}(\tau) \\
& \Gamma_{4,2}(\tau)= \frac{2}{25}\left[\bar{B}_{2} \frac{H_{2}(\tau)}{c}+\bar{A}_{2} c^{2} G_{2}(\tau)\right]-\frac{2}{297}\left[\bar{B}_{6} \frac{H_{6}(\tau)}{c^{5}}+\bar{A}_{6} c^{6} G_{6}(\tau)\right] \\
&-\frac{n(n-1)}{2(2 n+3)(2 n+1)^{2}}\left[\bar{B}_{n+2} \frac{H_{n+2}(\tau)}{c^{n+1}}+\bar{A}_{n+2} c^{n+2} G_{n+2}(\tau)\right] \\
& \Gamma_{n, 2}(\tau)= \frac{n(n-1)}{2(2 n-5)(2 n-3)^{2}}\left[\bar{B}_{n-2} \frac{H_{n-2}(\tau)}{c^{n-3}}+\bar{A}_{n-2} c^{n-2} G_{n-2}(\tau)\right] \\
& \Gamma_{4,4}(\tau)= \frac{5}{189} \bar{B}_{4} \frac{H_{6}(\tau)}{c^{5}}-\frac{\bar{A}_{4}}{175} c^{2} G_{2}(\tau) \\
& \Gamma_{n, 4}(\tau)= \frac{(n+1)(n+2)}{2(2 n-1)(2 n+1)^{2}} \bar{B}_{n} \frac{H_{n+2}(\tau)}{c^{n+1}}-\frac{(n-3)(n-2)}{2(2 n-1)(2 n-3)^{2}} \bar{A}_{n} c^{n-2} G_{n-2}(\tau) \\
& \Gamma^{3}(\tau)
\end{aligned}
$$

It is obvious, now, that as $c \rightarrow 0+$ the only terms that "survive" in Eq. (57) are those that are not multiplied by $c$, namely, $\left\{\Gamma_{n, 0}(\tau) G_{n}(\zeta) ; n=4,6, \ldots\right\}$. Let us denote by $\psi_{0}(\tau, \zeta)$ the expression composed of the aforementioned terms only, that is, 


$$
\begin{aligned}
\psi_{0}(\tau, \zeta) \equiv & \sum_{n=2,4, \ldots}^{\infty} \Gamma_{n, 0}(\tau) G_{n}(\zeta) \\
= & {\left[\frac{\bar{B}_{2}}{6} c G_{1}(\tau)+\frac{2 \bar{A}_{2}}{25} c^{4} G_{4}(\tau)+\bar{C}_{2} c^{2} G_{2}(\tau)+\bar{D}_{2} \frac{H_{2}(\tau)}{c}\right] G_{2}(\zeta) } \\
+ & {\left[\frac{-\bar{B}_{4}}{175} \frac{H_{2}(\tau)}{c}+\frac{\bar{A}_{4}}{189} c^{6} G_{6}(\tau)+\bar{C}_{4} c^{4} G_{4}(\tau)+\bar{D}_{4} \frac{H_{4}(\tau)}{c^{3}}\right] G_{4}(\zeta)+\cdots } \\
+ & {\left[\frac{-(n-3)(n-2)}{2(2 n-1)(2 n-3)^{2}} \bar{B}_{n} \frac{H_{n-2}}{c^{n-3}}\right.} \\
& +\frac{(n+1)(n+2)}{2(2 n-1)(2 n+1)^{2}} \bar{A}_{n} c^{n+2} G_{n+2}(\tau) \\
& \left.+\bar{C}_{n} c^{n} G_{n}(\tau)+\bar{D}_{n} \frac{H_{n}(\tau)}{c^{n-1}}\right] G_{n}(\zeta)+\cdots
\end{aligned}
$$

Note that if we take the limit, as the focal length vanishes, we obtain

$$
\psi_{0}^{*}(r, \theta)=\lim _{c \rightarrow 0+} \psi_{0}(\tau, \zeta)
$$

with

$$
\begin{aligned}
\psi_{0}^{*}(r, \theta)= & {\left[A_{2} r^{2}+B_{2} r^{-1}+C_{2} r^{4}+D_{2} r\right] G_{2}^{*}(\theta) } \\
& +\left[A_{4} r^{4}+B_{4} r^{-3}+C_{4} r^{6}+D_{4} r^{-1}\right] G_{4}^{*}(\theta)+\cdots \\
& +\left[A_{2 n} r^{2 n}+B_{2 n} r^{1-2 n}+C_{2 n} r^{2 n+2}+D_{2 n} r^{3-2 n}\right] G_{2 n}^{*}(\theta)+\cdots
\end{aligned}
$$

where

$$
G_{n}^{*}(\theta) \equiv G_{n}(\zeta) \quad\left[\text { e.g. }, G_{2}(\zeta)=\frac{1}{2}\left(1-\zeta^{2}\right)=\frac{1}{2}\left(1-\cos ^{2} \theta\right)=\frac{1}{2} \sin ^{2} \theta=G_{2}^{*}(\theta)\right],
$$

which is the general solution of Stokes flow in spherical coordinates (where the operator $E^{4}$ is completely separable).

Now, imposing the B.C.(5-8) on Eq. (58), we obtain

$$
\Gamma_{n, 0}(\tau)=0, \quad n=4,6,8, \ldots
$$

which implies

$$
\bar{A}_{n}=\bar{B}_{n}=\bar{C}_{n}=\bar{D}_{n}=0, \quad n=4,6,8, \ldots
$$

Consequently, Eq. (58) is reduced to

$$
\begin{aligned}
\psi_{0}(\tau, \zeta) & =\Gamma_{2,0}(\tau) G_{2}(\zeta) \\
& =\left[\frac{\bar{B}_{2}}{6} c G_{1}(\tau)+\bar{C}_{2} c^{2} G_{2}(\tau)+\frac{2}{25} \bar{A}_{2} c^{4} G_{4}(\tau)+\bar{D}_{2} \frac{H_{2}(\tau)}{c}\right] G_{2}(\zeta)
\end{aligned}
$$


Now, the solution (48) [or (57)] should agree with Eq. (62) in the limit, that is,

$$
\lim _{c \rightarrow 0+} \psi(\tau, \zeta)=\psi_{0}(\tau, \zeta)
$$

Since $\psi_{0}$ involves only $G_{2}(\zeta)$, we concentrate on the $\tau$-dependence of $\psi$ corresponding to $G_{2}(\zeta)$, namely, $g_{2}(\tau)$. From Eqs. (47) and (57) we have

$$
\begin{aligned}
g_{2}(\tau)= & \Gamma_{2,0}(\tau)+c^{2} \Gamma_{2,2}(\tau)+c^{4} \Gamma_{2,4}(\tau) \\
= & \frac{\bar{B}}{6} c G_{1}(\tau)+\bar{C}_{2} c^{2} G_{2}(\tau)+\left(\frac{2 \bar{A}_{2}}{25}-c^{2} \frac{\bar{A}_{4}}{175}\right) c^{4} G_{4}(\tau) \\
& +\bar{D}_{2} c^{-1} H_{2}(\tau)-c^{2}\left(\frac{\bar{B}_{4}}{175}-c^{2} \frac{2 \bar{B}_{2}}{25}\right) c^{-3} H_{4}(\tau) .
\end{aligned}
$$

We observe that for $c=0+$ the right-hand side of Eq. (64) agrees with the right-hand side of Eq. (62), as it should, since the two extra terms,

$$
c^{2} \frac{\bar{A}_{4}}{175} c^{4} G_{4}(\tau) \text { and } c^{2}\left(\frac{\bar{B}_{4}}{175}-c^{2} \frac{2 \bar{B}_{2}}{25}\right) c^{-3} H_{4}(\tau)
$$

are proportional to $c^{2}$. However, there is an important qualitative difference between these two terms, in that the second one introduces (for infinitesimal values of $c$ ) a type of dependence, $c^{-3} H_{4}(\tau) G_{2}(\zeta) \sim r^{-3} \sin ^{2} \theta$, which does not appear in the sphere-in-cell solution, neither should it appear for an infinitesimal perturbation. Accordingly, we set the coefficient $\mathscr{B}_{4}$ of $H_{4}(\tau) G_{2}(\zeta)$ in Eq. (48) equal to nil, obtaining

$$
\bar{B}_{4}=14 c^{2} \bar{B}_{2}, \quad \text { or } \quad B_{4}=14 B_{2} \quad\left(\mathscr{B}_{4}=0\right) .
$$

This is the restriction of the solution space imposed by our geometrical condition. Equation (65) provides the additional relation required to make the problem "well posed". The system of Eqs. (48), (53), (54), and (65) can be solved to obtain a unique solution in the form of a series, Eq. (47).

Determination of $g_{2}(\tau)$. From Eq. (48), using Eq. (65), we obtain

$$
g_{2}(\tau)=c^{2}\left[E_{1} G_{1}(\tau)+E_{2} G_{2}(\tau)+E_{3} G_{4}(\tau)+E_{4} H_{2}(\tau)\right],
$$

where

$$
E_{1}=\frac{1}{6} B_{2}, \quad E_{2}=c^{-2} C_{2}, \quad E_{3}=c^{-2} \mathscr{A}_{4}, \quad E_{4}=c^{-2} D_{2} .
$$

Applying the B.C.'s (53) we obtain

$$
g_{2}(\tau)=\frac{c^{2}}{D}\left[\Lambda_{2} G_{2}(\tau)+\Lambda_{3}\left(\frac{5 G_{4}\left(\tau_{\beta}\right)}{G_{1}\left(\tau_{\beta}\right)} G_{1}(\tau)+G_{4}(\tau)\right)+\Lambda_{4} H_{2}(\tau)\right],
$$


where the constants $D, \Lambda_{2}, \Lambda_{3}$, and $\Lambda_{4}$ are given by

$$
\begin{gathered}
D=\frac{1}{2 G_{2}\left(\tau_{\beta}\right)} \operatorname{det}\left[\begin{array}{ccc}
G_{2}\left(\tau_{\alpha}\right) & \left(G_{4}\left(\tau_{\alpha}\right)+\frac{5 \tau_{\alpha}}{\tau_{\beta}} G_{4}\left(\tau_{\beta}\right)\right) & H_{2}\left(\tau_{\alpha}\right) \\
G_{2}^{\prime}\left(\tau_{\alpha}\right) & \left(G_{4}^{\prime}\left(\tau_{\alpha}\right)+\frac{5}{\tau_{\beta}} G_{4}\left(\tau_{\beta}\right)\right) & H_{2}^{\prime}\left(\tau_{\alpha}\right) \\
G_{2}\left(\tau_{\beta}\right) & 6 G_{4}\left(\tau_{\beta}\right) & H_{2}\left(\tau_{\beta}\right)
\end{array}\right], \\
\Lambda_{2}=G_{4}\left(\tau_{\alpha}\right) H_{2}^{\prime}\left(\tau_{\alpha}\right)-G_{4}^{\prime}\left(\tau_{\alpha}\right) H_{2}\left(\tau_{\alpha}\right)-\frac{5}{\tau_{\beta}} G_{4}\left(\tau_{\beta}\right)\left[H_{2}\left(\tau_{\alpha}\right)-\tau_{\alpha} H_{2}^{\prime}\left(\tau_{\alpha}\right)\right], \\
\Lambda_{3}=G_{2}^{\prime}\left(\tau_{\alpha}\right) H_{2}\left(\tau_{\alpha}\right)-G_{2}\left(\tau_{\alpha}\right) H_{2}^{\prime}\left(\tau_{\alpha}\right)=-\frac{1}{2}, \\
\Lambda_{4}=G_{2}\left(\tau_{\alpha}\right) G_{4}^{\prime}\left(\tau_{\alpha}\right)-G_{2}^{\prime}\left(\tau_{\alpha}\right) G_{4}\left(\tau_{\alpha}\right)+\frac{5}{\tau_{\beta}} G_{4}\left(\tau_{\beta}\right)\left[G_{2}\left(\tau_{\alpha}\right)-\tau_{\alpha} G_{2}^{\prime}\left(\tau_{\alpha}\right)\right],
\end{gathered}
$$

where the primes denote the first derivatives of the corresponding functions. Equation (69) can also be written as

$$
D=\frac{1}{2 G_{2}\left(\tau_{\beta}\right)}\left[\Lambda_{2} G_{2}\left(\tau_{\beta}\right)+6 \Lambda_{3} G_{4}\left(\tau_{\beta}\right)+\Lambda_{4} H_{2}\left(\tau_{\beta}\right)\right] .
$$

At this level of approximation the solution is given by

$$
\psi(\tau, \zeta) \approx \psi^{(2)}(\tau, \zeta) \equiv g_{2}(\tau) G_{2}(\zeta)
$$

with $g_{2}(\tau)$ given by Eq. $(68)$; therefore,

$$
\psi^{(2)}(\tau, \zeta)=\frac{c^{2}}{D}\left[\Lambda_{2} G_{2}(\tau)+\Lambda_{3}\left(\frac{5 G_{4}\left(\tau_{\beta}\right)}{G_{1}\left(\tau_{\beta}\right)} G_{1}(\tau)+G_{4}(\tau)\right)+\Lambda_{4} H_{2}(\tau)\right] G_{2}(\zeta) .
$$

For convenience we list, here, the expressions for $G_{1}, G_{2}, G_{4}$, and $H_{2}$ :

$$
\begin{aligned}
& G_{1}(x)=-x, \\
& G_{2}(x)=\frac{1}{2}\left(1-x^{2}\right), \\
& G_{4}(x)=\frac{1}{8}\left(5 x^{2}-1\right)\left(1-x^{2}\right), \\
& H_{2}(x)=\frac{1}{4}\left(1-x^{2}\right) \ln \frac{x+1}{x-1}+\frac{x}{2},
\end{aligned}
$$

where $x=\tau$ or $\zeta$.

Sample streamlines around a prolate spheroid with $a_{3}=2\left(a_{1}=1\right)$ for the case that corresponds to a solid volume fraction $\gamma=0.05 \quad\left(c=\sqrt{3}, b_{1} \approx 3.283\right.$, $b_{3} \approx 3.712$ ) are given in Fig. 9 (see p. 178), based on Eq. (74). Numerical calculations in [6] indicate that $\psi^{(2)}(\tau, \zeta)$, as given by Eq. (74), contains most of the important physics of the flow, so long as $c$ is not too large. Sample streamlines around an oblate spheroid (see Appendix A) with $a_{3}=0.5$ for the case $\gamma=0.05$ are given in Fig. 10 (see p. 178).

Determination of the remaining $\tau$-functions of the expansion, namely, $g_{4}(\tau)$ and $g_{2 m}(\tau)$ for $m \geq 3$, is given in Appendix E. 


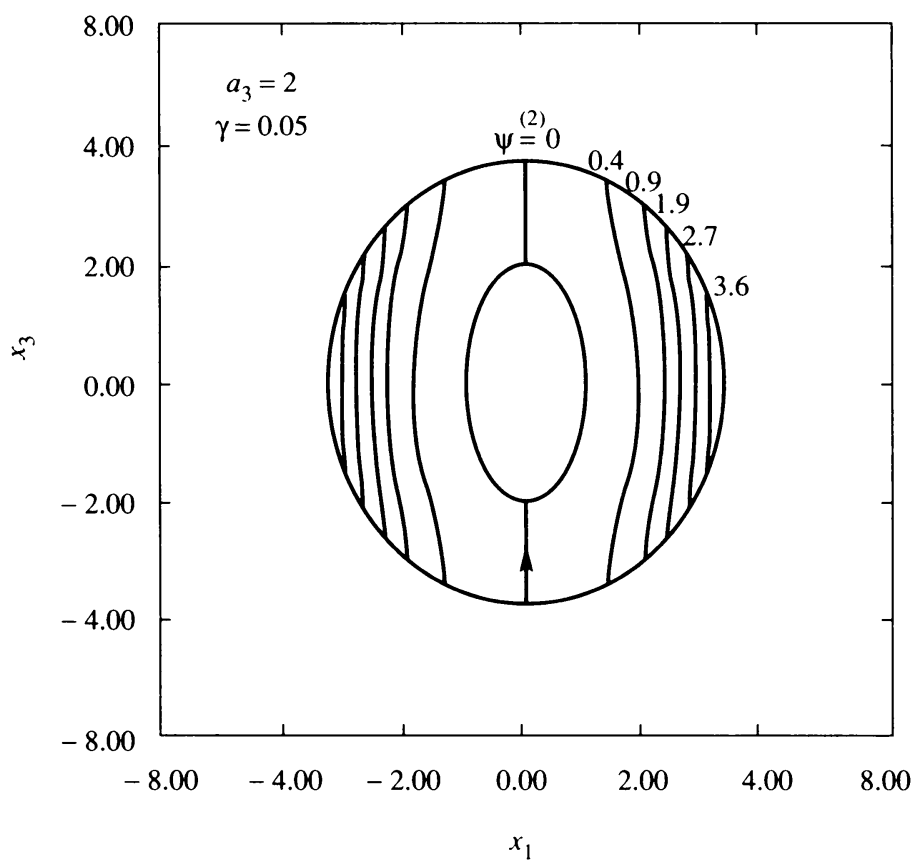

Fig. 9. Sample streamlines around a prolate spheroid-in-cell. The inner spheroid has major semiaxis $a_{3}=2$; the solid volume fraction value is $\gamma=0.05$.

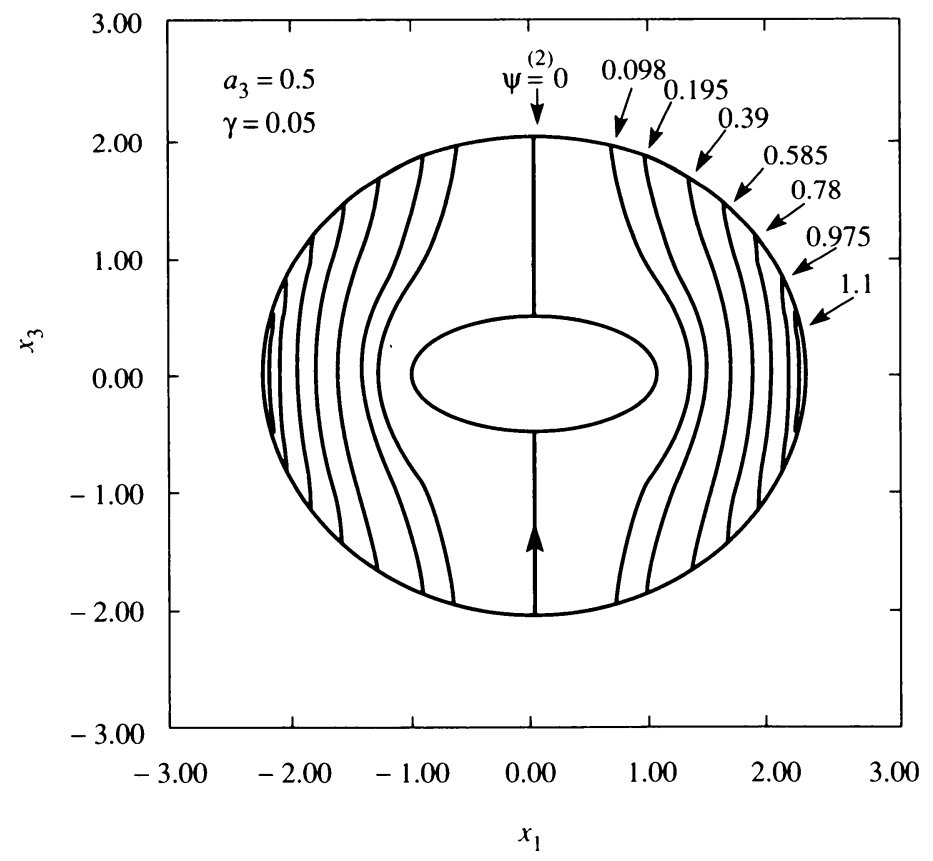

Fig. 10. Sample streamlines around an oblate spheroid-in-cell. The inner spheroid has minor semiaxis $a_{3}=0.5$; the solid volume fraction value is $\gamma=0.05$. 


\section{Conclusions.}

The complete solution of the equation for Stokes flow in spheroidal coordinates, $E^{4} \psi(\tau, \zeta)=0$, is obtained using the theory of generalized eigenfunctions, and the stream function is expressed as a sum of two functions-one from the 0-eigenspace, and one from the generalized 0-eigenspace of the operator $E^{2}$. The former function is expressed as an expansion in eigenfunctions of $E^{2}$, and the latter as an expansion in generalized eigenfunctions of $E^{2}$, Eq. (29). The generalized eigenfunctions of $E^{2}$ in spheroidal coordinates are determined here for the first time. Each generalized eigenfunction is expressed in terms of sums of products of Gegenbauer functions of mixed order [Eqs. (C.1)-(C.20), Appendix C]. Graphs of the streamlines of the basic flow fields represented by the first few generalized eigenfunctions are provided [Figs. 1-8]. A simplified series expansion of the stream function that produces regular, on the axis, velocity and vorticity fields is given explicitly [Eq. (30)].

Rearrangement of the terms of the expansion in eigenfunctions and generalized eigenfunctions, in such a way that terms having the same angular-type dependence $(\zeta$-dependence) are grouped together, produces an alternative expansion that is useful for the analysis of boundary-value problems [Eq. (31)]. This expansion is of a novel and interesting nature. The individual terms of this expansion are not solutions of $E^{4} \psi=0$, but the full expansion is. The $\tau$-dependence of $G_{n}(\zeta)$ in the generic term of this expansion is different from the $\tau$-dependence of $H_{n}(\zeta)$, and therefore the general solution is not separable in the usual sense. Nevertheless, the expansion exhibits some kind of separation, which we refer to as semiseparation.

The semiseparable solution is sufficiently general to handle interior, exterior, and spheroidal cell problems, with or without prescribed singularities on the axis of symmetry. A simplified version of the expansion that produces regular, on the axis, velocity and vorticity fields is also given [Eq. (32)]. This expansion is very useful for particular applications because of the orthogonality of the Gegenbauer functions of the first kind [Eq. (B.13)]. The form of semiseparable solutions causes problems with the simultaneous satisfaction of the equation and any generic set of boundary conditions. Hence, well posedness is not satisfied in the classical sense.

Finally, it is shown that, when the focal distance of the spheroidal system tends to nil, the semiseparable solution of Stokes flow in spheroidal coordinates, which resulted from the present work, reduces to the well-known separable solution in spherical coordinates.

A first application of the present theory in order to solve Stokes flow in a spheroidal cell (as a means of modelling flow through a swarm of spheroidal particles) is given as an example. Further development and utilization of this solution to obtain results for engineering applications (drag force and permeability) is given in a companion publication [6]. This application is a simple example of a problem that cannot be solved by an ad hoc technique but requires full use of the complete semiseparable solution. 


\section{Appendix A. Spheroidal geometry.}

A.1. Prolate Spheroidal Coordinates. The notation used in this work is consistent with Moon and Spencer [13] and Happel and Brenner [9].

Given a fixed positive number $c>0$, which we consider to be the semifocal distance of our system, we define the prolate spheroidal coordinates $(\eta, \theta, \varphi)$ as

$$
\begin{aligned}
& x_{1}=c \sinh \eta \sin \theta \cos \varphi, \\
& x_{2}=c \sinh \eta \sin \theta \sin \varphi, \\
& x_{3}=c \cosh \eta \cos \theta,
\end{aligned}
$$

where

$$
0 \leq \eta<+\infty, \quad 0 \leq \theta \leq \pi, \quad 0 \leq \varphi<2 \pi .
$$

The $(\eta, \theta, \varphi)$ system is orthogonal.

To every fixed value of $\eta \in(0,+\infty)$ there corresponds the unique prolate spheroid, Fig. 11,

$$
S_{\eta_{0}}: \frac{x_{1}^{2}+x_{2}^{2}}{c^{2} \sinh ^{2} \eta_{0}}+\frac{x_{3}^{2}}{c^{2} \cosh ^{2} \eta_{0}}=1
$$

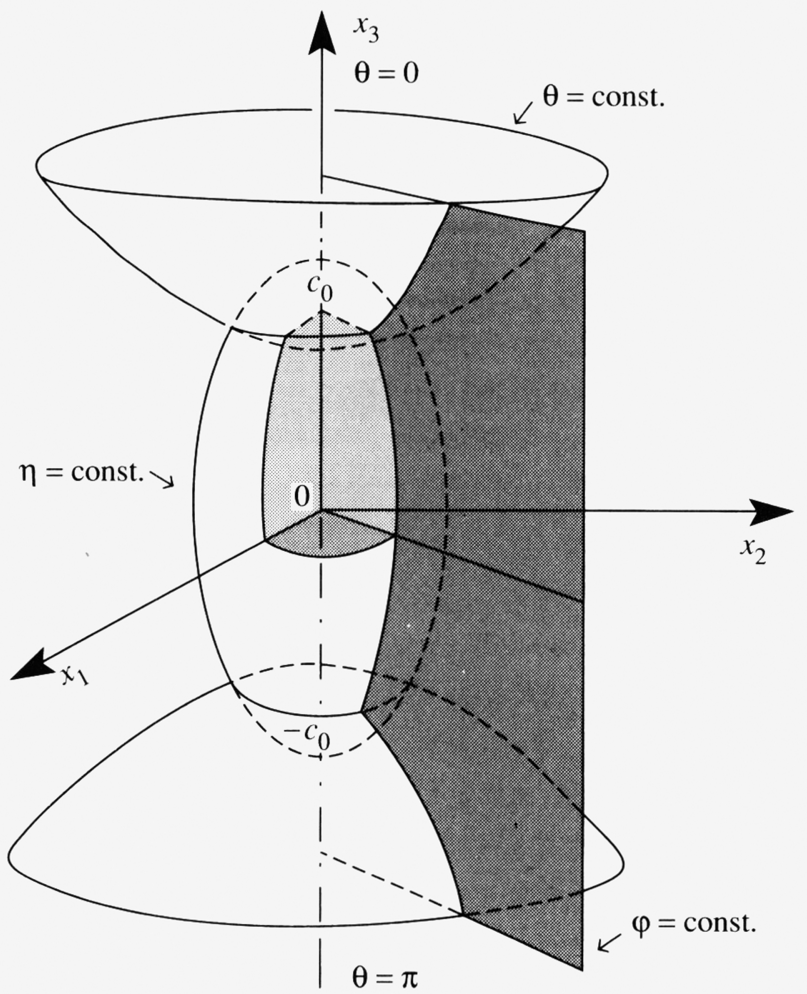

FIG. 11. System of prolate spheroidal coordinates 
with major semiaxis (on the axis of symmetry) $a_{3}(\eta)=c \cosh \eta$, minor semiaxis

$$
a_{1}(\eta)=c \sinh \eta \text { and eccentricity } \varepsilon(\eta)=\frac{1}{\cosh \eta} .
$$

In particular, as $\eta \rightarrow 0+$

$$
a_{3}(\eta) \rightarrow c, \quad a_{1}(\eta) \rightarrow 0, \quad \varepsilon(\eta) \rightarrow 1
$$

and the prolate spheroid $S_{\eta}$ degenerates to the focal line segment

$$
S_{0}:\{(0,0, t): t \in[-c,+c]\} .
$$

On the other hand, as $\eta \rightarrow+\infty$

$$
\varepsilon(\eta) \rightarrow 0 \quad\left[a_{3}(\eta)-a_{1}(\eta)\right] \rightarrow 0
$$

and the spheroid approaches a sphere located at infinity.

Following [9] we introduce the transformation

$$
\tau=\cosh \eta, \quad 1 \leq \tau<+\infty, \quad \zeta=\cos \theta, \quad-1 \leq \zeta \leq+1,
$$

which, of course, preserves the orthogonality of the system. Then, (A.1) reduces to

$$
\begin{aligned}
& x_{1}=c \sqrt{\tau^{2}-1} \sqrt{1-\zeta^{2}} \cos \varphi, \\
& x_{2}=c \sqrt{\tau^{2}-1} \sqrt{1-\zeta^{2}} \sin \varphi, \\
& x_{3}=c \tau \zeta,
\end{aligned}
$$

where

$$
1 \leq \tau<+\infty, \quad-1 \leq \zeta \leq+1, \quad 0 \leq \varphi<2 \pi .
$$

The generic spheroid (A.3) has the equation

$$
S_{\tau}: \frac{x_{1}^{2}+x_{2}^{2}}{c^{2}\left(\tau^{2}-1\right)}+\frac{x_{3}^{2}}{c^{2} \tau^{2}}=1
$$

with semiaxes

$$
a_{3}(\tau)=c \tau, \quad a_{1}(\tau)=c \sqrt{\tau^{2}-1},
$$

and eccentricity $\varepsilon(\tau)=1 / \tau$.

A.2. Oblate Spheroidal Coordinates. For oblate spheroidal coordinates the roles of $\sinh \eta$ and $\cosh \eta$ are interchanged. In this case the focal segment is replaced by the focal disc, since the axis of rotation is perpendicular to the line connecting the two focii. The equation of a generic oblate spheroid assumes the form

$$
\frac{x_{1}^{2}+x_{2}^{2}}{\bar{c}^{2} \cosh ^{2} \eta}+\frac{x_{3}^{2}}{\bar{c}^{2} \sinh ^{2} \eta}=1,
$$

where $\bar{c}$ denotes the semifocal distance of the spheroid. In terms of the variables

$$
\lambda=\sinh \eta, \quad \zeta=\cos \theta
$$


the oblate spheroidal coordinates are expressed as

$$
\begin{aligned}
& x_{1}=\bar{c} \sqrt{1+\lambda^{2}} \sqrt{1-\zeta^{2}} \cos \varphi, \\
& x_{2}=\bar{c} \sqrt{1+\lambda^{2}} \sqrt{1-\zeta^{2}} \sin \varphi, \\
& x_{3}=\bar{c} \lambda \zeta
\end{aligned}
$$

where

$$
0 \leq \lambda<+\infty, \quad-1 \leq \zeta \leq+1, \quad 0 \leq \varphi<2 \pi .
$$

The transformation (A.15) can also be obtained from Eq. (A.9) via the formal substitution

$$
\tau \rightarrow i \lambda, \quad c \rightarrow-i \bar{c} .
$$

Therefore, this simple substitution dictates the algorithm that translates results from the prolate to the oblate system. Note that the very same substitution should also be used to translate results from the oblate to the prolate system, that is,

$$
\lambda \rightarrow i \tau, \quad \bar{c} \rightarrow-i c,
$$

if the positivity of the expressions $c \sqrt{\tau^{2}-1}$ and $\bar{c} \sqrt{1+\lambda^{2}}$ is to be preserved. In the present work the analysis is done in the framework of prolate spheroidal coordinates. The foregoing substitution can be used, at any level, to obtain the corresponding expressions for oblate spheroids.

The metric coefficients in prolate geometry are

$$
h_{\eta}=c \sqrt{\tau^{2}-\zeta^{2}}, \quad h_{\theta}=c \sqrt{\tau^{2}-\zeta^{2}}, \quad h_{\varphi}=c \sqrt{\tau^{2}-1} \sqrt{1-\zeta^{2}} .
$$

Appendix B. Gegenbauer functions. The Gegenbauer functions of the first kind are polynomials of degree $n$, furnished by the formulae

$$
\begin{aligned}
& G_{0}(x)=1, \\
& G_{1}(x)=-x,
\end{aligned}
$$

and

$$
\begin{aligned}
G_{n}(x) & =\frac{1}{(2 n-1)}\left[P_{n-2}(x)-P_{n}(x)\right] \\
& =\frac{1}{(n-1) !} \frac{d^{n-2}}{d x^{n-2}}\left(\frac{x^{2}-1}{2}\right)^{n-1}
\end{aligned}
$$

for every $n \geq 2$, where $P_{n}(x)$ denotes the corresponding Legendre polynomial. The Gegenbauer functions of the second kind, with the exception of the zeroth and the first-order ones, are functions exhibiting logarithmic singularities on the axis of symmetry. They are given by

$$
\begin{aligned}
& H_{0}(x)=-x=G_{1}(x), \\
& H_{1}(x)=-1=-G_{0}(x),
\end{aligned}
$$


and

$$
\begin{aligned}
H_{n}(x) & =\frac{1}{(2 n-1)}\left[Q_{n-2}(x)-Q_{n}(x)\right] \\
& =\frac{1}{2} G_{n}(x) \begin{cases}\ln \frac{1+x}{1-x}+K_{n}(x), & |x|<1, \\
\ln \frac{x+1}{x-1}+K_{n}(x), & |x|>1\end{cases}
\end{aligned}
$$

for every $n \geq 2$, where

$$
K_{n}(x)=-\sum_{k=1}^{n / 2,(n+1) / 2} \frac{(2 n-4 k+1)}{(2 k-1)(n-k)}\left[1-\frac{(2 k-1)(n-k)}{n(n-1)}\right] G_{n-2 k+1}(x),
$$

and the summation terminates with the integer $n / 2$ or $n+\frac{1}{2}$, as $n$ runs through even or odd numbers, respectively. $Q_{n}(x)$ stands for the Legendre function of the second kind.

The logarithmic factors in Eq. (B.6) can also be expressed in terms of inverse hyperbolic functions as

$$
\begin{array}{ll}
\frac{1}{2} \ln \frac{1+x}{1-x}=\tanh ^{-1} x, & |x|<1, \\
\frac{1}{2} \ln \frac{x+1}{x-1}=\operatorname{coth}^{-1} x, & |x|>1 .
\end{array}
$$

Noting that the even-order Gegenbauer polynomials are even functions of $x$, while the odd-order ones are odd functions of $x$, the theory of telescopic series can be used to derive the relations

$$
\begin{aligned}
& G_{0}(x)=-H_{1}(x)=\sum_{n=1}^{\infty}(4 n-1) G_{2 n}(x), \\
& G_{1}(x)=H_{0}(x)=-\sum_{n=1}^{\infty}(4 n+1) G_{2 n+1}(x) .
\end{aligned}
$$

Indeed, by virtue of Eq. (B.3), both series (B.10) and (B.11) are telescopic series that converge for every $x \in(-1,+1)$, since

$$
\lim _{n \rightarrow \infty} P_{n}(x)=0, \quad x \in(-1,+1) .
$$

The limit (B.12) is a simple consequence of [17, Theorem 61]. Relations (B.10) and (B.11) imply that the Gegenbauer functions $G_{0}, G_{1}, H_{0}, H_{1}$ are linearly dependent on the Gegenbauer functions $G_{n}$ of order $n \geq 2$. The Gegenbauer functions of order $n \geq 2$ are linearly independent. Furthermore, the Gegenbauer polynomials $G_{n}(x)$ for $n \geq 2$ are orthogonal with respect to the inner product

$$
\begin{aligned}
\left(G_{n}(x), G_{m}(x)\right) & =\int_{-1}^{+1} \frac{G_{n}(x) G_{m}(x)}{1-x^{2}} d x \\
& =\frac{2}{n(n-1)(2 n-1)} \delta_{n m}, \quad n \geq 2, \quad m \geq 2,
\end{aligned}
$$

$\delta_{n m}$ being the Kronecker delta. 
The inner product (B.13) becomes singular for the set of functions $G_{0}(x), G_{1}(x)$, and $H_{n}(x), n \geq 0$.

In view of formulae (B.1)-(B.7) it is not hard to prove the following recurrence relations:

$$
\begin{aligned}
x^{2} G_{0}(x) & =G_{0}(x)-2 G_{2}(x), \\
x^{2} G_{1}(x) & =G_{1}(x)+2 G_{3}(x), \\
x^{2} G_{2}(x) & =\frac{1}{5} G_{2}(x)+\frac{4}{5} G_{4}(x), \\
x^{2} G_{3}(x) & =\frac{3}{7} G_{3}(x)+\frac{4}{7} G_{5}(x), \\
x^{2} G_{n}(x) & =\alpha_{n} G_{n-2}(x)+\gamma_{n} G_{n}(x)+\beta_{n} G_{n+2}(x)
\end{aligned}
$$

for $n \geq 4$, and

$$
\begin{aligned}
& x^{2} H_{0}(x)=x^{2} G_{1}(x) \\
& x^{2} H_{1}(x)=-x^{2} G_{0}(x) \\
& x^{2} H_{2}(x)=-\frac{1}{3} G_{1}(x)+\frac{1}{5} H_{2}(x)+\frac{4}{5} H_{4}(x), \\
& x^{2} H_{3}(x)=\frac{1}{15} G_{0}(x)+\frac{3}{7} H_{3}(x)+\frac{4}{7} H_{5}(x) \\
& x^{2} H_{n}(x)=\alpha_{n} H_{n-2}(x)+\gamma_{n} H_{n}(x)+\beta_{n} H_{n+2}(x)
\end{aligned}
$$

for $n \geq 4$, where

$$
\begin{aligned}
\alpha_{n} & =\frac{(n-3)(n-2)}{(2 n-3)(2 n-1)}, \quad n \geq 4, \\
\beta_{n} & =\frac{(n+1)(n+2)}{(2 n-1)(2 n+1)}, \quad n \geq 4, \\
\gamma_{n} & =\frac{n}{(n-3)} \alpha_{n}+\frac{(n-1)}{(n-2)} \beta_{n}=\frac{2 n^{2}-2 n-3}{(2 n+1)(2 n-3)}, \quad n \geq 4 .
\end{aligned}
$$

Appendix C. Generalized eigenfunctions of $E^{2}$ in spheroidal coordinates. The generalized eigenfunctions of $E^{2}$, see Eq. (20), are

$$
\begin{aligned}
& \Omega_{0}^{(1)}=-G_{0}(\tau) G_{2}(\zeta)-G_{2}(\tau) G_{0}(\zeta), \\
& \Omega_{0}^{(2)}=\frac{1}{3} G_{0}(\tau) G_{3}(\zeta)-G_{2}(\tau) G_{1}(\zeta), \\
& \Omega_{0}^{(3)}=\frac{1}{3} G_{3}(\tau) G_{0}(\zeta)-G_{1}(\tau) G_{2}(\zeta), \\
& \Omega_{0}^{(4)}=\frac{1}{3} G_{1}(\tau) G_{3}(\zeta)+\frac{1}{3} G_{3}(\tau) G_{1}(\zeta), \\
& \Omega_{1}^{(1)}=\Omega_{0}^{(4)}, \\
& \Omega_{1}^{(2)}=-\Omega_{0}^{(3)}, \\
& \Omega_{1}^{(3)}=-\Omega_{0}^{(2)}, \\
& \Omega_{1}^{(4)}=\Omega_{0}^{(1)},
\end{aligned}
$$




$$
\begin{aligned}
& \Omega_{2}^{(1)}=\frac{2}{25} G_{2}(\tau) G_{4}(\zeta)+\frac{2}{25} G_{4}(\tau) G_{2}(\zeta), \\
& \Omega_{2}^{(2)}=\frac{2}{25} G_{2}(\tau) H_{4}(\zeta)+\frac{2}{25} G_{4}(\tau) H_{2}(\zeta)+\frac{1}{6} G_{2}(\tau) G_{1}(\zeta), \\
& \Omega_{2}^{(3)}=\frac{2}{25} H_{2}(\tau) G_{4}(\zeta)+\frac{2}{25} H_{4}(\tau) G_{2}(\zeta)+\frac{1}{6} G_{1}(\tau) G_{2}(\zeta), \\
& \Omega_{2}^{(4)}=\frac{2}{25} H_{2}(\tau) H_{4}(\zeta)+\frac{2}{25} H_{4}(\tau) H_{2}(\zeta)+\frac{1}{6} G_{1}(\tau) H_{2}(\zeta)+\frac{1}{6} H_{2}(\tau) G_{1}(\zeta), \\
& \Omega_{3}^{(1)}=\frac{2}{49} G_{3}(\tau) G_{5}(\zeta)+\frac{2}{49} G_{5}(\tau) G_{3}(\zeta), \\
& \Omega_{3}^{(2)}=\frac{2}{49} G_{3}(\tau) H_{5}(\zeta)+\frac{2}{49} G_{5}(\tau) H_{3}(\zeta)-\frac{1}{90} G_{3}(\tau) G_{0}(\zeta), \\
& \Omega_{3}^{(3)}=\frac{2}{49} H_{3}(\tau) G_{5}(\zeta)+\frac{2}{49} H_{5}(\tau) G_{3}(\zeta)-\frac{1}{90} G_{0}(\tau) G_{3}(\zeta), \\
& \Omega_{3}^{(4)}=\frac{2}{49} H_{3}(\tau) H_{5}(\zeta)+\frac{2}{49} H_{5}(\tau) H_{3}(\zeta)-\frac{1}{90} G_{0}(\tau) H_{3}(\zeta)-\frac{1}{90} H_{3}(\tau) G_{0}(\zeta)
\end{aligned}
$$

and for $n=4,5, \ldots$

$$
\begin{aligned}
\Omega_{n}^{(1)}= & -\frac{\alpha_{n}}{2(2 n-3)}\left[G_{n-2}(\tau) G_{n}(\zeta)+G_{n}(\tau) G_{n-2}(\zeta)\right] \\
& +\frac{\beta_{n}}{2(2 n+1)}\left[G_{n+2}(\tau) G_{n}(\zeta)+G_{n}(\tau) G_{n+2}(\zeta)\right], \\
\Omega_{n}^{(2)}= & -\frac{\alpha_{n}}{2(2 n-3)}\left[G_{n-2}(\tau) H_{n}(\zeta)+G_{n}(\tau) H_{n-2}(\zeta)\right] \\
& +\frac{\beta_{n}}{2(2 n+1)}\left[G_{n+2}(\tau) H_{n}(\zeta)+G_{n}(\tau) H_{n+2}(\zeta)\right], \\
\Omega_{n}^{(3)}= & -\frac{\alpha_{n}}{2(2 n-3)}\left[H_{n-2}(\tau) G_{n}(\zeta)+H_{n}(\tau) G_{n-2}(\zeta)\right] \\
& +\frac{\beta_{n}}{2(2 n+1)}\left[H_{n+2}(\tau) G_{n}(\zeta)+H_{n}(\tau) G_{n+2}(\zeta)\right], \\
\Omega_{n}^{(4)}= & -\frac{\alpha_{n}}{2(2 n-3)}\left[H_{n-2}(\tau) H_{n}(\zeta)+H_{n}(\tau) H_{n-2}(\zeta)\right] \\
& +\frac{\beta_{n}}{2(2 n+1)}\left[H_{n+2}(\tau) H_{n}(\zeta)+H_{n}(\tau) H_{n+2}(\zeta)\right],
\end{aligned}
$$

where the coefficients $\alpha_{n}$ and $\beta_{n}$ are given by Eqs. (B.24) and (B.25), respectively.

Appendix D. $\tau$-Dependent functions of the expansion (31). The $\tau$-dependent functions $g_{n}(\tau)$ and $h_{n}(\tau)$ are summarized as

$$
\begin{aligned}
g_{0}(\tau)= & \left(A_{0}^{1}-A_{1}^{4}\right) G_{0}(\tau)+\left(A_{0}^{3}-A_{1}^{2}\right) G_{1}(\tau) \\
& -\left(B_{0}^{1}+B_{1}^{4}\right) G_{2}(\tau)+\frac{1}{3}\left(B_{0}^{3}-B_{1}^{2}-\frac{B_{3}^{2}}{30}\right) G_{3}(\tau)-\frac{B_{3}^{4}}{90} H_{3}(\tau), \\
g_{1}(\tau)= & \left(A_{0}^{2}-A_{1}^{3}\right) G_{0}(\tau)+\left(A_{0}^{4}+A_{1}^{1}\right) G_{1}(\tau) \\
& -\left(B_{0}^{2}-B_{1}^{3}-\frac{B_{2}^{2}}{6}\right) G_{2}(\tau)+\frac{B_{2}^{4}}{6} H_{2}(\tau)-\frac{1}{3}\left(B_{0}^{4}+B_{1}^{1}\right) G_{3}(\tau),
\end{aligned}
$$




$$
\begin{aligned}
g_{2}(\tau)= & A_{2}^{1} G_{2}(\tau)+A_{2}^{3} H_{2}(\tau)-\left(B_{0}^{1}+B_{1}^{4}\right) G_{0}(\tau) \\
& -\left(B_{0}^{3}-B_{1}^{2}-\frac{B_{2}^{3}}{6}\right) G_{1}(\tau) \\
& +\frac{1}{25}\left(2 B_{2}^{1}-\frac{B_{4}^{1}}{7}\right) G_{4}(\tau)+\frac{1}{25}\left(2 B_{2}^{3}-\frac{B_{4}^{3}}{7}\right) H_{4}(\tau) \\
g_{3}(\tau)= & A_{3}^{1} G_{3}(\tau)+A_{3}^{3} H_{3}(\tau)+\frac{1}{3}\left(B_{0}^{2}-B_{1}^{3}-\frac{B_{3}^{3}}{30}\right) G_{0}(\tau) \\
& +\frac{1}{3}\left(B_{0}^{4}+B_{1}^{1}\right) G_{1}(\tau)+\frac{1}{49}\left(2 B_{3}^{1}-\frac{B_{5}^{1}}{3}\right) G_{5}(\tau)+\frac{1}{49}\left(2 B_{3}^{3}-\frac{B_{5}^{3}}{3}\right) H_{5}(\tau) \\
& \quad+\frac{1}{2(2 n-3)}\left(\beta_{n-2} B_{n-2}^{1}-\alpha_{n} B_{n}^{1}\right) G_{n-2}(\tau) \\
& +\frac{1}{2(2 n-3)}\left(\beta_{n-2} B_{n-2}^{3}-\alpha_{n} B_{n}^{3}\right) H_{n-2}(\tau) \\
& +\frac{1}{2(2 n+1)}\left(\beta_{n} B_{n}^{1}-\alpha_{n+2} B_{n+2}^{1}\right) G_{n+2}(\tau) \\
& +\frac{1}{2(2 n+1)}\left(\beta_{n} B_{n}^{3}-\alpha_{n+2} B_{n+2}^{3}\right) H_{n+2}(\tau)
\end{aligned}
$$

for $n=4,5,6, \ldots$,

$$
\begin{aligned}
h_{2}(\tau)= & A_{2}^{2} G_{2}(\tau)+A_{2}^{4} H_{2}(\tau) \\
& +\frac{B_{2}^{4}}{6} G_{1}(\tau)+\frac{1}{25}\left(2 B_{2}^{2}-\frac{B_{4}^{2}}{7}\right) G_{4}(\tau)+\frac{1}{25}\left(2 B_{2}^{4}-\frac{B_{4}^{4}}{7}\right) H_{4}(\tau), \\
h_{3}(\tau)= & A_{3}^{2} G_{3}(\tau)+A_{3}^{4} H_{3}(\tau) \\
& -\frac{B_{3}^{4}}{90} G_{0}(\tau)+\frac{1}{49}\left(2 B_{3}^{2}-\frac{B_{5}^{2}}{3}\right) G_{5}(\tau)+\frac{1}{49}\left(2 B_{3}^{4}-\frac{B_{5}^{4}}{3}\right) H_{5}(\tau),
\end{aligned}
$$

and

$$
\begin{aligned}
h_{n}(\tau)= & A_{n}^{2} G_{n}(\tau)+A_{n}^{4} H_{n}(\tau) \\
& +\frac{1}{2(2 n-3)}\left(\beta_{n-2} B_{n-2}^{2}-\alpha_{n} B_{n}^{2}\right) G_{n-2}(\tau) \\
& +\frac{1}{2(2 n-3)}\left(\beta_{n-2} B_{n-2}^{4}-\alpha_{n} B_{n}^{4}\right) H_{n-2}(\tau) \\
& +\frac{1}{2(2 n+1)}\left(\beta_{n} B_{n}^{2}-\alpha_{n+2} B_{n+2}^{2}\right) G_{n+2}(\tau) \\
& +\frac{1}{2(2 n+1)}\left(\beta_{n} B_{n}^{4}-\alpha_{n+2} B_{n+2}^{4}\right) H_{n+2}(\tau)
\end{aligned}
$$

for $n=4,5,6, \ldots$. 
Appendix E. Determination of $g_{4}(\tau)$ and $g_{2 m}(\tau)$ for $m \geq 3$.

Determination of $g_{4}(\tau)$. From Eq. (48), using Eq. (65), we have

$$
g_{4}(\tau)=\mathscr{A}_{4} G_{2}(\tau)+\mathscr{A}_{6} G_{6}(\tau)+\mathscr{B}_{6} H_{6}(\tau)+C_{4} G_{4}(\tau)+D_{4} H_{4}(\tau),
$$

where $\mathscr{A}_{4}$ is already known from the previous step as

$$
\mathscr{A}_{4}=c^{2} \frac{\Lambda_{3}}{D}=-\frac{c^{2}}{2 D}
$$

Applying the B.C.'s (54) we obtain

$$
g_{4}(\tau)=\mathscr{A}_{4} G_{2}(\tau)+\frac{1}{D^{(4)}}\left[\Lambda_{2}^{(4)} G_{6}(\tau)+M_{2}^{(4)} H_{6}(\tau)+\Lambda_{0}^{(4)} G_{4}(\tau)+M_{0}^{(4)} H_{4}(\tau)\right],
$$

where $D^{(4)}, \Lambda_{2}^{(4)}, M_{2}^{(4)}, \Lambda_{0}^{(4)}, M_{0}^{(4)}$ are the determinants

$$
\begin{gathered}
D^{(4)}=\left[\begin{array}{llll}
G_{6}\left(\tau_{\alpha}\right) & H_{6}\left(\tau_{\alpha}\right) & G_{4}\left(\tau_{\alpha}\right) & H_{4}\left(\tau_{\alpha}\right) \\
G_{6}^{\prime}\left(\tau_{\alpha}\right) & H_{6}^{\prime}\left(\tau_{\alpha}\right) & G_{4}^{\prime}\left(\tau_{\alpha}\right) & H_{4}^{\prime}\left(\tau_{\alpha}\right) \\
G_{6}\left(\tau_{\beta}\right) & H_{6}\left(\tau_{\beta}\right) & G_{4}\left(\tau_{\beta}\right) & H_{4}\left(\tau_{\beta}\right) \\
30 G_{6}\left(\tau_{\beta}\right) & 30 H_{6}\left(\tau_{\beta}\right) & 12 G_{4}\left(\tau_{\beta}\right) & 12 H_{4}\left(\tau_{\beta}\right)
\end{array}\right], \\
\Lambda_{2}^{(4)}=-\mathscr{A}_{4}\left[\begin{array}{llll}
G_{2}\left(\tau_{\alpha}\right) & H_{6}\left(\tau_{\alpha}\right) & G_{4}\left(\tau_{\alpha}\right) & H_{4}\left(\tau_{\alpha}\right) \\
G_{2}^{\prime}\left(\tau_{\alpha}\right) & H_{6}^{\prime}\left(\tau_{\alpha}\right) & G_{4}^{\prime}\left(\tau_{\alpha}\right) & H_{4}^{\prime}\left(\tau_{\alpha}\right) \\
G_{2}\left(\tau_{\beta}\right) & H_{6}\left(\tau_{\beta}\right) & G_{4}\left(\tau_{\beta}\right) & H_{4}\left(\tau_{\beta}\right) \\
G_{2}\left(\tau_{\beta}\right) & 30 H_{6}\left(\tau_{\beta}\right) & 12 G_{4}\left(\tau_{\beta}\right) & 12 H_{4}\left(\tau_{\beta}\right)
\end{array}\right], \\
M_{2}^{(4)}=-\mathscr{A}_{4}\left[\begin{array}{llll}
G_{6}\left(\tau_{\alpha}\right) & G_{2}\left(\tau_{\alpha}\right) & G_{4}\left(\tau_{\alpha}\right) & H_{4}\left(\tau_{\alpha}\right) \\
G_{6}^{\prime}\left(\tau_{\alpha}\right) & G_{2}^{\prime}\left(\tau_{\alpha}\right) & G_{4}^{\prime}\left(\tau_{\alpha}\right) & H_{4}^{\prime}\left(\tau_{\alpha}\right) \\
G_{6}\left(\tau_{\beta}\right) & G_{2}\left(\tau_{\beta}\right) & G_{4}\left(\tau_{\beta}\right) & H_{4}\left(\tau_{\beta}\right) \\
30 G_{6}\left(\tau_{\beta}\right) & G_{2}\left(\tau_{\beta}\right) & 12 G_{4}\left(\tau_{\beta}\right) & 12 H_{4}\left(\tau_{\beta}\right)
\end{array}\right], \\
\Lambda_{0}^{(4)}=-\mathscr{A}_{4}\left[\begin{array}{llll}
G_{6}\left(\tau_{\alpha}\right) & H_{6}\left(\tau_{\alpha}\right) & G_{2}\left(\tau_{\alpha}\right) & H_{4}\left(\tau_{\alpha}\right) \\
G_{6}^{\prime}\left(\tau_{\alpha}\right) & H_{6}^{\prime}\left(\tau_{\alpha}\right) & G_{2}^{\prime}\left(\tau_{\alpha}\right) & H_{4}^{\prime}\left(\tau_{\alpha}\right) \\
G_{6}\left(\tau_{\beta}\right) & H_{6}\left(\tau_{\beta}\right) & G_{2}\left(\tau_{\beta}\right) & H_{4}\left(\tau_{\beta}\right) \\
30 G_{6}\left(\tau_{\beta}\right) & 30 H_{6}\left(\tau_{\beta}\right) & G_{2}\left(\tau_{\beta}\right) & 12 H_{4}\left(\tau_{\beta}\right)
\end{array}\right], \\
M_{0}^{(4)}=-\mathscr{A}_{4}\left[\begin{array}{llll}
G_{6}\left(\tau_{\alpha}\right) & H_{6}\left(\tau_{\alpha}\right) & G_{4}\left(\tau_{\alpha}\right) & G_{2}\left(\tau_{\alpha}\right) \\
G_{6}^{\prime}\left(\tau_{\alpha}\right) & H_{6}^{\prime}\left(\tau_{\alpha}\right) & G_{4}^{\prime}\left(\tau_{\alpha}\right) & G_{2}^{\prime}\left(\tau_{\alpha}\right) \\
G_{6}\left(\tau_{\beta}\right) & H_{6}\left(\tau_{\beta}\right) & G_{4}\left(\tau_{\beta}\right) & G_{2}\left(\tau_{\beta}\right) \\
30 G_{6}\left(\tau_{\beta}\right) & 30 H_{6}\left(\tau_{\alpha}\right) & 12 G_{4}\left(\tau_{\beta}\right) & G_{2}\left(\tau_{\beta}\right)
\end{array}\right] .
\end{gathered}
$$

Determination of $g_{2 m}(\tau)$ for $m \geq 3$. From Eq. (48) we obtain

$$
\begin{aligned}
g_{2 m}(\tau)= & \mathscr{A}_{2 m} G_{2 m-2}(\tau)+\mathscr{B}_{2 m} H_{2 m-2}(\tau)+\mathscr{A}_{2 m+2} G_{2 m+2}(\tau) \\
& +\mathscr{B}_{2 m+2} H_{2 m+2}(\tau)+C_{2 m} G_{2 m}(\tau)+D_{2 m} H_{2 m}(\tau),
\end{aligned}
$$


where $\mathscr{A}_{2 m}$ and $\mathscr{B}_{2 m}$ are known from the previous step. Applying the B.C.s (65) and solving for the unknowns $\mathscr{A}_{2 m+2}, \mathscr{B}_{2 m+2}, \mathscr{C}_{2 m}$, and $\mathscr{D}_{2 m}$ we obtain

$$
\begin{aligned}
g_{2 m}(\tau)= & \mathscr{A}_{2 m} G_{2 m-2}(\tau)+\mathscr{B}_{2 m} H_{2 m-2}(\tau) \\
& +\frac{1}{D^{(2 m)}}\left[\Lambda_{2}^{(2 m)} G_{2 m+2}(\tau)+M_{2}^{(2 m)} H_{2 m+2}(\tau)\right. \\
& \left.+\Lambda_{0}^{(2 m)} G_{2 m}(\tau)+M_{0}^{(2 m)} H_{2 m}(\tau)\right],
\end{aligned}
$$

where $D^{(2 m)}, \Lambda_{2}^{(2 m)}, M_{2}^{(2 m)}, \Lambda_{0}^{(2 m)}$, and $M_{0}^{(2 m)}$ are the determinants defined as

$$
\begin{aligned}
& D^{(2 m)}=\left[\begin{array}{llll}
G_{2 m+2}\left(\tau_{\alpha}\right) & H_{2 m+2}\left(\tau_{\alpha}\right) & G_{2 m}\left(\tau_{\alpha}\right) & H_{2 m}\left(\tau_{\alpha}\right) \\
G_{2 m+2}^{\prime}\left(\tau_{\alpha}\right) & H_{2 m+2}^{\prime}\left(\tau_{\alpha}\right) & G_{2 m}^{\prime}\left(\tau_{\alpha}\right) & H_{2 m}^{\prime}\left(\tau_{\alpha}\right) \\
G_{2 m+2}\left(\tau_{\beta}\right) & H_{2 m+2}\left(\tau_{\beta}\right) & G_{2 m}\left(\tau_{\beta}\right) & H_{2 m}\left(\tau_{\beta}\right) \\
(2 m+2)(2 m+1) & (2 m+2)(2 m+1) & 2 m(2 m-1) & 2 m(2 m-1) \\
\cdot G_{2 m+2}\left(\tau_{\beta}\right) & \cdot H_{2 m+2}\left(\tau_{\beta}\right) & \cdot G_{2 m}\left(\tau_{\beta}\right) & \cdot H_{2 m}\left(\tau_{\beta}\right)
\end{array}\right], \\
& \Lambda_{2}^{(2 m)}=-\left[\begin{array}{clll}
\mathscr{A}_{2 m} G_{2 m-2}\left(\tau_{\alpha}\right)+\mathscr{B}_{2 m} H_{2 m-2}\left(\tau_{\alpha}\right) & H_{2 m+2}\left(\tau_{\alpha}\right) & G_{2 m}\left(\tau_{\alpha}\right) & H_{2 m}\left(\tau_{\alpha}\right) \\
\mathscr{2}_{2 m} G_{2 m-2}^{\prime}\left(\tau_{\alpha}\right)+\mathscr{B}_{2 m} H_{2 m-2}^{\prime}\left(\tau_{\alpha}\right) & H_{2 m+2}^{\prime}\left(\tau_{\alpha}\right) & G_{2 m}^{\prime}\left(\tau_{\alpha}\right) & H_{2 m}^{\prime}\left(\tau_{\alpha}\right) \\
\mathscr{A}_{2 m} G_{2 m-2}\left(\tau_{\beta}\right)+\mathscr{B}_{2 m} H_{2 m-2}\left(\tau_{\beta}\right) & H_{2 m+2}\left(\tau_{\beta}\right) & G_{2 m}\left(\tau_{\beta}\right) & H_{2 m}\left(\tau_{\beta}\right) \\
(2 m-2)(2 m-3)\left(\mathscr{A}_{2 m} G_{2 m-2}\left(\tau_{\beta}\right)\right. & (2 m+2)(2 m+1) & 2 m(2 m-1) & 2 m(2 m-1) \\
\left.+\mathscr{B}_{2 m} H_{2 m-2}\left(\tau_{\beta}\right)\right) & \cdot H_{2 m+2}\left(\tau_{\beta}\right) & \cdot G_{2 m}\left(\tau_{\beta}\right) & \cdot H_{2 m}\left(\tau_{\beta}\right)
\end{array}\right] \\
& M_{2}^{(2 m)}=-\left[\begin{array}{lclc}
G_{2 m+2}\left(\tau_{\alpha}\right) & \mathscr{A}_{2 m} G_{2 m-2}\left(\tau_{\alpha}\right) & G_{2 m}\left(\tau_{\alpha}\right) & H_{2 m}\left(\tau_{\alpha}\right) \\
& +\mathscr{B}_{2 m} H_{2 m-2}\left(\tau_{\alpha}\right) & G_{2 m}^{\prime}\left(\tau_{\alpha}\right) & H_{2 m}^{\prime}\left(\tau_{\alpha}\right) \\
G_{2 m+2}^{\prime}\left(\tau_{\alpha}\right) & \mathscr{A}_{2 m} G_{2 m-2}^{\prime}\left(\tau_{\alpha}\right) & G_{2 m}^{\prime}\left(\tau_{\alpha}\right. & \\
& +\mathscr{B}_{2 m} H_{2 m-2}^{\prime}\left(\tau_{\alpha}\right) & & \\
G_{2 m+2}\left(\tau_{\beta}\right) & \mathscr{A}_{2 m} G_{2 m-2}\left(\tau_{\beta}\right) & G_{2 m}\left(\tau_{\beta}\right) & H_{2 m}\left(\tau_{\beta}\right) \\
& +\mathscr{B}_{2 m} H_{2 m-2}\left(\tau_{\beta}\right) & & \\
(2 m+2)(2 m+1) & (2 m-2)(2 m-3) & 2 m(2 m-1) & 2 m(2 m-1) \\
\cdot G_{2 m+2}\left(\tau_{\beta}\right) & \cdot\left[\mathscr{A}_{2 m} G_{2 m-2}\left(\tau_{\beta}\right)\right. & \cdot G_{2 m}\left(\tau_{\beta}\right) & \cdot H_{2 m}\left(\tau_{\beta}\right) \\
& \left.+B_{2 m} H_{2 m-2}\left(\tau_{\beta}\right)\right] & &
\end{array}\right]
\end{aligned}
$$

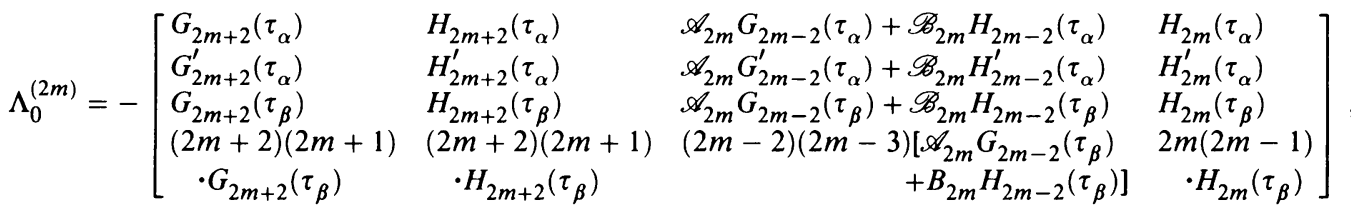

and

$$
M_{0}^{(2 m)}=-\left[\begin{array}{lllr}
G_{2 m+2}\left(\tau_{\alpha}\right) & H_{2 m+2}\left(\tau_{\alpha}\right) & G_{2 m}\left(\tau_{\alpha}\right) & \mathscr{A}_{2 m} G_{2 m-2}\left(\tau_{\alpha}\right)+B_{2 m} H_{2 m+2}\left(\tau_{\alpha}\right) \\
G_{2 m+2}^{\prime}\left(\tau_{\alpha}\right) & H_{2 m+2}^{\prime}\left(\tau_{\alpha}\right) & G_{2 m}^{\prime}\left(\tau_{\alpha}\right) & \mathscr{A}_{2 m} G_{2 m-2}^{\prime}\left(\tau_{\alpha}\right)+\mathscr{B}_{2 m} H_{2 m+2}^{\prime}\left(\tau_{\alpha}\right) \\
G_{2 m+2}\left(\tau_{\beta}\right) & H_{2 m+2}\left(\tau_{\beta}\right) & G_{2 m}\left(\tau_{\beta}\right) & \mathscr{A}_{2 m} G_{2 m-2}\left(\tau_{\beta}\right)+\mathscr{B}_{2 m} H_{2 m+2}\left(\tau_{\beta}\right) \\
(2 m+2)(2 m+1) & (2 m+2)(2 m+1) & 2 m(2 m-1) & (2 m-2)(2 m-3)\left[\mathscr{A}_{2 m} G_{2 m-2}\left(\tau_{\beta}\right)\right. \\
\cdot G_{2 m+2}\left(\tau_{\beta}\right) & \cdot H_{2 m+2}\left(\tau_{\beta}\right) & \cdot G_{2 m}\left(\tau_{\beta}\right) & \left.+\mathscr{B}_{2 m} H_{2 m-2}\left(\tau_{\beta}\right)\right]
\end{array}\right] .
$$


Acknowledgments. We thank Mr. D. Lymberopoulos and Dr. C. Paraskeva for their help in preparing the figures.

\section{Appendix F. Notation.}

$$
\begin{aligned}
& A_{n}^{i} \text { constant coefficients, Eq. (16) } \\
& A_{n}, \bar{A}_{n}^{n} \text { constants, and corresponding scaled constants, Eq. (56) } \\
& \mathscr{A}_{n} \text { arbitrary constants defined by Eq. (49) } \\
& a_{1} \text { minor (major) semiaxis of the prolate (oblate) spheroid } \\
& a_{3} \text { major (minor) semiaxis of the prolate (oblate) spheroid } \\
& B_{n}^{i} \text { constant coefficients, Eq. (19) } \\
& \mathscr{B}_{n} \text { arbitrary constants defined by Eq. (50) } \\
& B_{n}, \bar{B}_{n} \text { constants, and corresponding scaled constants, Eq. (56) } \\
& b_{3}, b_{1} \text { dimensionless major (minor) semiaxis and minor (major) semiaxis } \\
& \text { of the external prolate (oblate) spheroid, respectively } \\
& C_{n}, \bar{C}_{n} \text { constants, and corresponding scaled constants, Eq. (56) } \\
& c \text { semifocal length of prolate spheroidal coordinates } \\
& \bar{c} \text { semifocal length of oblate spheroidal coordinates } \\
& D \text { determinant defined by Eq. (69) } \\
& D_{n}, \bar{D}_{n} \text { constants, and corresponding scaled constants, Eq. (56) } \\
& D^{(2 m)} \text { determinants defined by Eqs. (E.4), (E.11) } \\
& E^{2} \text { operator, Eq. (6) } \\
& E_{1}, E_{2}, E_{3}, E_{4} \text { constants } \\
& G_{n}(x) \text { Gegenbauer function of the first kind, of degree }\left(-\frac{1}{2}\right) \\
& \text { and of order } n \\
& g_{n}(\tau) \text { functions of } \tau \text {, Eq. (31) } \\
& H_{n}(x) \text { Gegenbauer function of the second kind, of degree }\left(-\frac{1}{2}\right) \\
& \text { and of order } n \\
& h_{n}(\tau) \text { functions of } \tau \text {, Eq. (31) } \\
& h_{\eta}, h_{\theta}, h_{\varphi} \text { metric coefficients, Eq. (A.19) } \\
& K_{n}(x) \text { functions defined by Eq. (B.7) } \\
& \hat{\mathbf{n}} \text { outward unit normal vector } \\
& P_{n}(x) \text { Legendre function of the first kind, of order } n \\
& Q_{n}(x) \text { Legendre function of the second kind, of order } n \\
& S_{\alpha} \text { surface of the internal (solid) spheroid of the spheroid-in-cell } \\
& S_{\beta} \text { surface of the external spheroid of the spheroid-in-cell } \\
& S_{\eta}, S_{\tau} \text { spheroidal surface for } \eta=\text { const. , } \tau=\text { const., respectively } \\
& r \text { spherical radial coordinate } \\
& \tilde{u} \text { dimensional approach velocity; in the spheroid-in-cell model } \\
& v \text { dimensionless velocity } \\
& v_{\tau}, v_{\zeta} \quad \tau \text { and } \zeta \text { components of } \mathbf{v} \\
& x_{1}, x_{2}, x_{3} \text { Cartesian coordinates }
\end{aligned}
$$

Greek Letters.

$\alpha$ parameter defined by Eq. (42)

$\alpha_{n}$ coefficient given by Eq. (B.24) 
$\beta$ parameter defined by Eq. (42)

$\beta_{n}$ coefficient given by Eq. (B.25)

$\Gamma_{n}^{i}$ constant coefficients, Eq. (40)

$\Gamma_{i j}(\tau)$ functions of $\tau$, Eq. (57) and (57.1-9)

$\gamma$ solid volume fraction of the spheroid-in-cell

$\gamma_{n}$ coefficients, Eq. (B.26)

$\Delta_{n}^{i}$ constant coefficients, Eq. (41)

$\delta_{n m}$ Kronecker delta

$\varepsilon$ eccentricity

$\zeta$ modified spheroidal coordinate, Eq. (A.8)

$\hat{\zeta}$ unit vector in the direction of the axis $\zeta$

$\eta$ spheroidal coordinate

$\hat{\eta}$ unit vector in the $\eta$-direction

$\Theta_{n}^{(i)}(\tau, \zeta)$ eigenfunctions of $E^{2}$, Eqs. (12) to (15)

$\theta$ spheroidal coordinate

$\lambda$ modified oblate spheroidal coordinate, Eq. (A.14)

$\tau$ modified prolate spheroidal coordinate, Eq. (A.8)

$\hat{\boldsymbol{\tau}}$ unit vector in the direction of the axis $\tau$

$\varphi$ spheroidal coordinate

$\hat{\boldsymbol{\varphi}}$ unit vector in the direction of the axis $\varphi$

$\psi(\tau, \zeta)$ stream function, Eq. (7)

$\tilde{\psi}(\tau, \zeta)$ any function that satisfies Eq. (18)

$\psi^{(2)}(\tau, \zeta)$ leading term of $\psi$, Eq. (74)

$\psi_{0}^{*}(r, \theta)$ general solution of Stokes flow in spherical coordinates, Eq. (59.2)

$\Omega_{n}^{(i)}(\tau, \zeta)$ generalized eigenfunctions of $E^{2}$, defined by Eq. (20) and given by Eqs. (C.1)-(C.20)

\section{REFERENCES}

[1] A. T. Chwang, Hydromechanics of Low-Reynolds-Number Flow. Part 3, Motion of Spheroidal Particle in Quadratic Flows, J. Fluid Mech. 72, 17-34 (1975)

[2] A. T. Chwang and T. Y. Wu, Hydromechanics of Low-Reynolds-Number Flow. Part 1. Rotation of Axisymmetric Prolate Bodies, J. Fluid Mech. 63, 607-622 (1974)

[3] A. T. Chwang and T. Y. Wu, Hydromechanics of Low-Reynolds-Number Flow. Part 2. Singularity Method for Stokes Flows, J. Fluid Mech. 67, 787-815 (1975)

[4] A. T. Chwang and T. Y. Wu, Hydromechanics of Low-Reynolds-Number Flow. Part 4. Translation of Spheroids, J. Fluid Mech. 75, 677-689 (1976)

[5] T. Dabros, A Singularity Method for Calculating Hydrodynamic Forces and Particle Velocities in Low-Reynolds-Number Flows, J. Fluid Mech 156, 1-21 (1985)

[6] G. Dassios, M. Hadjinicolaou, and A. C. Payatakes, On Stokes flow through a swarm of spheroidal particles, submitted (1991)

[7] S. Goldstein, Modern Developments in Fluid Dynamics, Clarendon Press, Oxford, 1938, pp. 114115

[8] J. Happel, Viscous Flow in Multiparticle Systems: Slow Motion of Fluids Relative to Beds of Spherical Particles, A. I. Ch. E. J. 4, 197-201 (1958)

[9] J. Happel and H. Brenner, Low Reynolds Number Hydrodynamics, Prentice Hall, Englewood Cliffs, NJ, 1965; and Martinus Nijholl Publishers, Dordrecht, 1986

[10] D. J. Jeffrey and Y. Onishi, Calculation of the Resistance and Mobility Functions for Two Unequal Rigid Spheres in Low-Reynolds Number Flow, J. Fluid Mech 139, 261-290 (1985) 
[11] S. Kuwabara, The Forces Experienced by Randomly Distributed Parallel Circular Cylinders or Spheres in a Viscous Flow at Small Reynolds Numbers, J. Phys. Soc. Japan 14, 527-532 (1959)

[12] H. Lamb, Hydrodynamics, Dover, New York, 1932, pp. 604-605

[13] P. Moon and D. E. Spencer, Field Theory Handbook, Springer-Verlag, New York, 1961, pp. 28-29

[14] P. M. Morse and H. Feshbach, Methods of Theoretical Physics, Vols. I, II, McGraw-Hill, New York, 1953, pp. 547-549, 600-604

[15] A. Oberbeck, Ueber Stationäre Flüssigkeitsbewegungen mit Berücksichtigung der inneren Reibung, J. Reine Angew. Math. 81, 62-80 (1876)

[16] L. E. Payne and W. H. Pell, The Stokes Flow Problem for a Class of Axially Symmetric Bodies, J. Fluid Mech. 7, 529-549 (1960)

[17] E. R. Rainville, Special Functions, Chelsea, New York, 1971, p. 173

[18] J. M. Rallison and A. Acrivos, A Numerical Study of the Deformation and Burst of a Viscous Drop in an Extensional Flow, J. Fluid Mech. 89, 191-200 (1978)

[19] R. A. Sampson, On Stoke's Current Function, Philos. Trans. Roy. Soc. London Ser. A 182, 449-518 (1891)

[20] M. Stimson and G. B. Jeffery, The Motion of Two-Spheres in a Viscous Fluid, Proc. Roy. Soc. A 111, $110-116(1926)$

[21] B. J. Yoon and S. Kim, A Boundary Collocation Method for the Motion of Two Spheroids in Stokes Flows: Hydrodynamic and Colloidal Interactions, Internat. J. Multiphase Flow 16, 639-649 (1990) 